\title{
Preference Conditions for Invertible Demand Functions*
}

\author{
Theodoros M. Diasakos \\ University of Stirling
}

\author{
Georgios Gerasimou \\ University of St Andrews
}

August 20, 2020

\begin{abstract}
It is frequently assumed in several domains of economics that demand functions are invertible in prices. At the primitive level of preferences, however, the corresponding characterization has remained elusive. We identify necessary and sufficient conditions on a utility-maximizing consumer's preferences for her demand function to be continuous and invertible: strict convexity, strict monotonicity and differentiability in the sense of Rubinstein (2006). We further show that Rubinstein differentiability is equivalent to the indifference sets being smooth, which is weaker than Debreu's (1972) notion of preference smoothness. We finally discuss implications of our analysis for demand functions that satisfy the "strict law of demand".
\end{abstract}

${ }^{*}$ We are grateful to Hugo Sonnenschein, Phil Reny and the anonymous referees for very useful comments. Any errors are our own. 


\section{Introduction}

Invertibility of demand is frequently assumed in several domains of economic inquiry that include consumer and revealed preference theory (Afriat, 2014; Matzkin and Ricther, 1991; Chiappori and Rochet, 1987; Cheng, 1985), the estimation of discrete or continuous demand systems that may be non-separable and non-parametric (Berry, Ghandhi, and Haile, 2013), portfolio choice (Kübler and Polemarchakis, 2017), general equilibrium theory (Hildenbrand, 1994), and industrial organization (Amir, Erickson, and Jin, 2017). In some of this work (e.g. Berry, Ghandi, and Haile, 2013; Cheng, 1985) the interest has naturally been on conditions that ensure invertibility of the relevant demand function/system. Focusing on a general neoclassical consumer-theoretic domain, the present paper goes one step further and contributes to this large literature by providing the first complete characterization of classes of preference relations that generate consumer demand functions that are invertible in prices.

Certain smoothness conditions on either the demand system directly (e.g. Gale and Nikaido, 1965) or -closer to our analysis- on the utility function that generates it (e.g. Katzner, 1970) have been known for a long time to be sufficient for invertibility. However, the more foundational question of whether it is also possible to identify conditions on a consumer's preferences that are simultaneously necessary and sufficient for invertibility has remained unanswered. Perhaps surprisingly, our main result shows that invertibility of a utility-maximizing consumer's demand function is characterized by three simple and behaviourally interpretable textbook conditions on the preferences that generate it: strict convexity, strict monotonicity and differentiability in the sense of Rubinstein (2006). The latter notion intuitively requires that for every bundle in the consumption set there exists a vector -which we refer to as the preference gradient- such that any arbitrarily small movement away from the bundle is in a direction that results to an improvement for the consumer if and only if the move is evaluated as positive by the vector.

Our characterization pertains to invertible demand functions generated by continuous preference relations and has two building blocks. The first establishes that strict convexity and strict monotonicity by themselves jointly characterize rational demand functions that have the onto/surjectivity property whereby for every bundle in the consumption set there are some -possibly non-unique- prices that rationalize the consumer's choice of that bundle. This result is of independent interest and although it involves relatively standard arguments, we have been unable to find a statement of it in the literature. The second building block establishes that, for onto demand functions in this class, the one-to-one/injectivity property is equivalent to Rubinstein differentiability of the preference relation that generates them. Proving this second part -and especially that preference differentiability is implied by injectivity- is not as straightforward and requires the use of novel arguments.

As we demonstrate by example, within the class of continuous, strictly convex and strictly monotonic preferences, the behaviourally interpretable notion of Rubinstein differentiability is weaker than the requirement that the preferences admit a smooth or even differentiable utility representation. In fact, Rubinstein differentiability turns out to be equivalent to a notion of weakly smooth preferences due to Neilson (1991). This generalizes the original notion of smooth preferences due to Debreu (1972) -which is equivalent to the existence of a smooth utility representation- by restricting attention to what happens within any given indifference set, not along the entire indifference relation itself.

From the applications' point of view, our result provides transparency in -and guidance for-applied work that assumes demand functions that are invertible in prices. As is often the case in practice, the 
analyst may assume an invertible demand function directly. Our characterization clarifies that, at the more primitive level of the generating preferences, the analyst effectively assumes strict convexity, strict monotonicity and an intuitive notion of differentiability that is weaker than anything that could guarantee proper differentiability of the utility function. In this sense our analysis shows that micro-founding a model that features invertible demand can be done by imposing the relevant necessary and sufficient structure directly on preferences, thereby avoiding unnecessarily strong assumptions and maximizing the model's domain of application. In particular, the expanded class of preferences that we show can generate invertible demands includes many that are representable by utility functions which are defined in a piecewise but non-smooth fashion and, for example, obey homotheticity in some regions but not in others, thus allowing for the consumer's marginal rates of substitution to change discontinuously even as the quantity of all goods increases by the same proportion. This expansion adds to the toolkit of the applied economist interested in analysing consumer behaviour when preferences may change -possibly non-smoothly- as income varies.

As an additional note of motivation for our contribution let us now recall that invertibility of the aggregate demand function in a pure exchange economy is equivalent to uniqueness of Walrasian equilibrium relative prices. As pointed out in Jerison and Quah (2008) and Hildenbrand (1994), for example, when the Walrasian market demand function satisfies the "strict law of demand" whereby the vectors of changes in prices and demanded quantities go in strictly opposite directions following a price change, the above invertibility condition is satisfied and the equilibrium is unique and stable. Our characterization contributes to the behavioural foundations of this analysis as it implies that if an individual (respectively, market) onto demand function satisfies the strict law of demand, then the consumer's (respectively, the representative consumer's, if one exists) preferences are necessarily strictly convex, strictly monotonic and differentiable. This implication makes a non-obvious step in the direction of fully characterizing preference structures generating demand functions that satisfy the strict law of demand, which remains an open problem.

The remainder of the paper is structured as follows. Section 2 states and decomposes the main result, and also illustrates it with two examples. The penultimate part of that section focuses on the special case where preferences are, in addition, homothetic or quasi-linear, and the final part discusses the implication of our analysis for the behavioural origins of the strict law of demand. Section 3 presents the proofs of all results that are stated in the main body of the paper. The Online Appendix provides additional material and results, including domain generalizations and the proofs of various non-obvious claims that we make in passing while informally discussing some aspects and implications of the results that are stated in the main body of the paper.

\section{Main Result, Decomposition and Implications}

We consider a consumption set $X$ that is an open and convex weak subset of $\mathbb{R}_{++}^{n}$. For two consumption bundles $x$ and $y$ in $X$ we write $x \geq y$ and $x \gg y$ whenever $x_{i} \geq y_{i}$ and $x_{i}>y_{i}$ for all $i \leq n$, respectively. We also write $x>y$ whenever $x \geq y$ and $x \neq y$. The consumer's preferences are captured by a continuous weak order $\succsim$ on $X$, i.e. by a complete and transitive binary relation whose graph is a closed subset of $X \times X$. Such preferences are convex if, for all $x, y \in X$ and any $\alpha \in[0,1], x \succsim y$ implies 
$\alpha x+(1-\alpha) y \succsim y$, and monotonic if $x \gg y$ implies $x \succ y$. They are strictly convex if, for all $x, y \in X$ and $\alpha \in(0,1), x \succsim y$ implies $\alpha x+(1-\alpha) y \succ y$, and strictly monotonic if $x>y$ implies $x \succ y$. For any $x \in X$ we let

$$
\mathcal{U}_{x}:=\{z \in X: z \succsim x\} \text { and } \mathcal{I}_{x}:=\{z \in X: z \sim x\}
$$

denote the weak upper-contour and indifference sets of $x$, respectively. For $A \subseteq X$, we let

$$
\max _{\succsim} A:=\{x \in A: x \succsim y \text { for all } y \in A\}
$$

denote the set of all $\succsim$-greatest elements in $A$. Given some set $Y \subseteq \mathbb{R}_{++}^{n}$ of income-normalized strictly positive prices, the budget correspondence $B: Y \rightarrow X$ is defined by

$$
B(p):=\{x \in X: p x \leq 1\},
$$

where the dot-product $p \cdot x$ for any $p, x \in \mathbb{R}^{k}$ and $1<k \leq n$ will be denoted simply by $p x$ throughout the paper. We will say that $\succsim$ generates the demand correspondence $x: Y \rightarrow X$ if the latter is defined by

$$
x(p):=\max _{\succsim} B(p) .
$$

We will refer to such a demand correspondence as rational. ${ }^{1}$ A rational demand correspondence is surjective or onto if, for all $x \in X$ there exists $p \in Y$ such that $x \in x(p)$. If $x(\cdot)$ is single-valued (hence a demand function), it is said to be injective or one-to-one if for all $p, p^{\prime} \in Y, p \neq p^{\prime}$ implies $x(p) \neq x\left(p^{\prime}\right)$. A demand function $x: Y \rightarrow X$ that is both injective and surjective is bijective or invertible. If $x(\cdot)$ has this property, then the inverse demand given by

$$
p(x):=\{p \in Y: x=x(p)\}
$$

is itself a well-defined bijective function $p: X \rightarrow Y$.

In addition to the standard properties of preferences that were introduced above, the problem under investigation naturally invites the introduction of some notion of preference differentiability or smoothness. The first notion of preference smoothness in the literature was proposed in Debreu (1972), where a preference relation $\succsim$ on a consumption set $X$ was defined to be smooth of order $r$, or $C^{r}$ for short, if the graph of the indifference relation -that is, the set $\{(x, y) \in X \times X: x \sim y\} \subset X \times X$-is a $C^{r}$-manifold on $X \times X .^{2}$ Debreu (1972) showed that a monotonic preference relation on $X$ is $C^{r}$ if and only if it is representable by a utility function that itself is $C^{r}$, or, equivalently, $r$ times continuously differentiable. Generalizing Debreu's notion, Neilson (1991) defined a preference relation on such a set $X$ as weakly

\footnotetext{
${ }^{1}$ Even though the budget correspondence remains nonempty- and convex-valued in our framework, it is no longer compactvalued because we assume that $X \subseteq \mathbb{R}_{++}^{n}$. It is therefore no longer an immediate consequence of standard results such as the Maximum Theorem that the demand correspondence generated by $\succsim$ is well-defined. Nevertheless, as we explain below, the preference structure that we consider turns out to be sufficiently strong to overcome this technical difficulty because it ensures that only interior consumption bundles will ever be demanded.

${ }^{2}$ Letting $A \subseteq \mathbb{R}^{n}$, a function $f: A \rightarrow \mathbb{R}^{n}$ is an homeomorphism if it is injective, continuous, and its inverse function is continuous on $f(A)$. Given an open $A \subseteq \mathbb{R}^{n}$, a $C^{r}$ function $f: A \rightarrow \mathbb{R}^{n}$ is a $C^{r}$ diffeomorphism if it is an homeomorphism with a $C^{r}$ inverse function. A set $M \subseteq \mathbb{R}^{n}$ is a $C^{r} k$-dimensional $(k \leq n)$ manifold if for every $x \in M$ there is a $C^{r}$ diffeomorphism $f: A \rightarrow \mathbb{R}^{n}$ ( $A \subseteq \mathbb{R}^{n}$ open) which carries the open set $A \cap\left(\mathbb{R}^{k} \times\left\{\boldsymbol{0}^{n-k}\right\}\right)$ onto an open neighborhood of $x$ in $M$. For more details and some economic-theoretic examples, see Chapter 1.H in Mas-Colell (1985).
} 
smooth of order $r$, or weakly $C^{r}$, if all its indifference sets $\mathcal{I}_{x}$ are $C^{r}$-manifolds on $X$. Neilson (1991) established that this notion of weak smoothness suffices for the resulting Hicksian demand function to be smooth. We will refer to preferences that are smooth in Neilson's sense simply as weakly smooth.

More recently, Rubinstein (2006) defined the preference relation $\succsim$ on $X$ to be differentiable if for every $x \in X$ there exists $p_{x} \in \mathbb{R}^{n} \backslash\{\mathbf{0}\}$ that makes the equality

$$
\left\{z \in \mathbb{R}^{n}: p_{x} \cdot z>0\right\}=\left\{z \in \mathbb{R}^{n}: \text { there exists } \lambda_{z}^{*}>0 \text { such that } x+\lambda z \succ x \text { for all } \lambda \in\left(0, \lambda_{z}^{*}\right)\right\}
$$

true. To see the intuition, note first that for distinct bundles $x$ and $z$ in $X$, Rubinstein (2006) defined $z$ to be an improvement direction at $x$ if there exists $\lambda^{*}>0$ such that $x+\lambda z \succ x$ for all $\lambda \in\left(0, \lambda^{*}\right)$, assuming $(x+\lambda z) \in X$. In words, a not necessarily positive vector $z$ is an improvement direction at bundle $x$ if "steering" $x$ towards $z$ by adding a sufficiently small amount of $z$ to $x$ results in a new consumption bundle that is strictly preferred to $x$. In light of this definition, the right hand side of (1) defines the set of all improvement directions at $x$. The left hand side of (1) on the other hand defines the set of all directions $z$ that are evaluated as strictly positive by some vector $p_{x}$ that depends on $x$. Thus, Rubinstein differentiability of preferences $\succsim$ at bundle $x$ requires the existence of a vector $p_{x}$ that makes the set of all directions that are evaluated as strictly positive by $p_{x}$ coincide with the set of all improvement directions of $\succsim$. If it exists, such a vector $p_{x}$ will be referred to as a preference gradient at $x$. An intuitive interpretation for the entries of $p_{x}$ is that they represent the consumer's "subjective values" of the different goods relative to the reference bundle $x$ : "Starting from $x$, any small move in a direction that is evaluated by this vector as positive is an improvement" (Rubinstein, 2006 p. 71). We note, finally, that this author also showed that, under strict convexity and strict monotonicity of $\succsim$, partial differentiability of a utility function that represents $\succsim$ also implies differentiability of that relation.

With the requisite concepts in place, our main result can now be formally stated.

Theorem 1 The following are equivalent for a continuous weak order $\succsim$ on $X$ :

(i). $\succsim$ is strictly convex, strictly monotonic and differentiable.

(ii). $\succsim$ is strictly convex, strictly monotonic and weakly smooth.

(iii). There is a unique, open set $Y \subseteq \mathbb{R}_{++}^{n}$ and a unique, continuous, invertible demand function $x: Y \rightarrow X$ that is generated by $\succsim$.

The statement of the theorem is a combination of Propositions 1-3, which are presented separately in the next section. To give the reader a better understanding of the interaction between the three preference axioms and the onto and one-to-one properties of the generated demand function, we decompose the theorem into its constituent parts and provide an outline of the relevant formal arguments. Before turning to this, however, an informal overview of our analysis might be instructive.

First, the problem of finding prices that rationalize the demand of a given consumption bundle is, naturally, a supporting-hyperplane existence question. Continuity and convexity of preferences in our environment do indeed ensure that such a hyperplane exists. Strict convexity, moreover, guarantees uniqueness of the demanded bundle at these prices, while strict monotonicity ensures that all prices are strictly positive. Rubinstein differentiability moreover implies that the (normalized) supporting prices are unique. This relatively straightforward argument establishes the part of Theorem 1 that claims the sufficiency of the postulated properties on preferences for the demand function to be invertible. It is 
not obvious that such a demand function is continuous, however, and a more involved argument is deployed to show that continuity of the demand function does indeed follow from preference differentiability. Even more challenging, finally, is to establish the part of the theorem that claims that invertibility of the demand function necessitates that the generating preference relation is differentiable. This amounts to showing that a given notion of smoothness for the functional representation of the graph of the indifference sets corresponds to the appropriate notion of smoothness for the preference relation. This turns out to be a rather abstract and non-trivial task.

\subsection{Characterization of Onto Demand Functions}

The first part of our decomposition characterizes onto demand functions by means of continuity, strict convexity and strict monotonicity alone.

Proposition 1 The following are equivalent for a continuous weak order $\succsim$ on X:

(i). $\succsim$ is strictly convex and strictly monotonic.

(ii). There is a set $Y \subseteq \mathbb{R}_{++}^{n}$ and an onto demand function $x: Y \rightarrow X$ that is generated by $\succsim$.

Although much easier to establish compared to the characterization of one-to-one demand functions that we offer later on, this result is of independent interest and, to our knowledge, novel. To outline briefly the intuition behind it, we introduce some additional definitions and notation, which will also be useful below. For any $A \subset \mathbb{R}^{n}$, we say that $p \in \mathbb{R}^{n} \backslash\{\mathbf{0}\}$ supports $A$ at $x$ if $p x \leq p z$ for any $z \in A$, and that $p$ supports $A$ at $x$ properly if $p x<p z$ for any $z \in A \backslash\{x\}$. Take now any $x \in X$. Given continuity and strict convexity, by the supporting hyperplane theorem there exists $p \in \mathbb{R}^{n} \backslash\{\mathbf{0}\}$ that supports $\mathcal{U}_{x}$ at $x$, and hence that $x \in \max _{\succsim}\{z \in X: p z \leq p x\}$. Given also strict monotonicity, any such $p$ must, in fact, be a strictly positive price vector. Therefore, defining the set $Y$ by

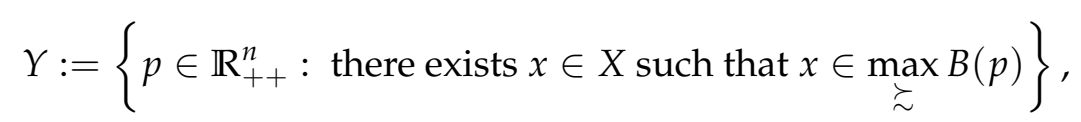

the mapping $x: Y \rightarrow X$ that is constructed by $x(p)=\max _{\succsim} B(p)$ is an onto demand correspondence. By strict convexity, moreover, this must actually be an onto demand function. Conversely, if $x: Y \rightarrow X$ is an onto demand function, then strict monotonicity of $\succsim$ readily follows from the strict positivity of prices. In addition, as $x(\cdot)$ is single-valued and generated by continuous and strictly monotonic preferences, strict convexity of $\succsim$ follows by the equivalence result in Bilancini and Boncinelli (2010): if a rational demand correspondence is generated by a strictly monotonic and continuous weak order, then the former is single-valued if and only if the latter is strictly convex.

\subsection{Characterization of (Continuous) Invertible Demand Functions}

To examine the second part of our decomposition, for arbitrary $x \in X$ consider first the projection of the indifference set $\mathcal{I}_{x}$ along the $i$ th dimension of $\mathbb{R}_{+}^{n}$,

$$
\mathcal{I}_{x}^{i}:=\left\{z_{i} \in \mathbb{R}_{+}: \text {there exists } z_{-i} \in \mathbb{R}_{+}^{n-1} \text { such that } z \in \mathcal{I}_{x}\right\},
$$

and define the set

$$
\mathcal{I}_{x}^{-i}:=\left\{z_{-i} \in \mathbb{R}_{+}^{n-1}: \text { there exists } z_{i} \in \mathbb{R}_{+} \text {such that } z \in \mathcal{I}_{x}\right\}
$$


analogously as the projection of $\mathcal{I}_{x}$ on $\mathbb{R}_{+}^{n-1}$, the resulting subspace when the $i$ th dimension is removed from $\mathbb{R}_{+}^{n}$. We can then construct the indifference-projection correspondence $l_{i}(\cdot \mid x): \mathcal{I}_{x}^{-i} \rightarrow \mathcal{I}_{x}^{i}$ for good $i$ by requiring

$$
z_{i} \in l_{i}\left(z_{-i} \mid x\right) \Longleftrightarrow z \in \mathcal{I}_{x}
$$

and observe that the graph of this correspondence is the indifference set $\mathcal{I}_{x}$. As we show in the Online Appendix, the mapping $l_{i}(\cdot \mid x)$ in our framework is actually a function that is locally convex and thus also continuous. ${ }^{3}$ As a result, its local subdifferential $\partial l_{i}\left(z_{-i} \mid x\right)$ which comprises the collection of the function's local subgradients ${ }^{4}$ at $z_{-i}$ is non-empty and fundamentally linked to its smoothness: $l_{i}(\cdot \mid x)$ is differentiable at $z_{-i}$ if and only if $\partial l_{i}\left(z_{-i} \mid x\right)$ is a singleton, in which case the unique local subgradient coincides with the gradient. With regard to interpretation, when $l_{i}(\cdot \mid x)$ is differentiable at $z_{-i}$ the entry $\partial l_{i}\left(z_{-i} \mid x\right) / \partial z_{j}$ of the gradient $\nabla l_{i}\left(z_{-i} \mid x\right)$ defines the marginal rate of substitution of good $i$ for good $j \neq i$. Indeed, by the Implicit Function Theorem (see also Lemma 5.3 in the Online Appendix), we have

$$
\frac{\partial l_{i}\left(z_{-i} \mid x\right)}{\partial z_{j}}=-\frac{\frac{\partial u(z)}{\partial z_{j}}}{\frac{\partial u(z)}{\partial z_{i}}}
$$

as long as there exists a utility function $u: X \rightarrow \mathbb{R}$ that represents $\succsim$ and is continuously differentiable at $z$. The right-hand side of this equation depicts the textbook definition of the marginal rate of substitution of good $i$ for good $j$, which results though by invoking the Implicit Function Theorem and thus assumes that the utility function is smooth by being at least continuously differentiable. By contrast, as we establish below, the left-hand side of (2) exists in a more general environment where preferences are strictly convex, strictly monotonic and differentiable.

Proposition 2 Suppose that the onto demand function $x: Y \rightarrow X$ for some $Y \subseteq \mathbb{R}_{++}^{n}$ is generated by the continuous weak order $\succsim$ on $X$. For any $x \in X$, the following are equivalent:

(i). For some $i \leq n, l_{i}(\cdot \mid x)$ is differentiable at $x_{-i}$.

(ii). $\succsim$ is differentiable at $x$.

(iii). $p(x)$ is a singleton.

(iv). For all $i \leq n, l_{i}(\cdot \mid x)$ is differentiable at $x_{-i}$.

Therefore, an onto demand function that is generated by a strictly convex and strictly monotonic continuous weak order $\succsim$ on $X$ is also injective and hence invertible if and only if $\succsim$ is differentiable. Upon letting $q_{-i}(x)$ denote the gradient (equivalently, the unique local subgradient) of the indifference-projection function $l_{i}(\cdot \mid x)$ for good $i \leq n$ at $x$, the preference gradient $p_{x}$ coincides with $p(x)$, the inverse demand at this bundle, and is determined by

$$
\begin{aligned}
q_{-i}(x) & =\nabla l_{i}\left(x_{-i} \mid x\right) \\
q_{i}(x) & =\frac{1}{x_{i}-q_{-i}(x) \cdot x_{-i}} \\
p(x) & =\left(q_{i}(x),-q_{i}(x) q_{-i}(x)\right),
\end{aligned}
$$

\footnotetext{
${ }^{3}$ Notice that, for any $x, z \in X$ with $z \sim x$, the mappings $l_{i}(\cdot \mid x)$ and $l_{i}(\cdot \mid z)$ coincide.

${ }^{4}$ We refer the reader to Section 4 of the Online Appendix for some background on local subgradients and subdifferentials.
} 
where $q_{-i}(x) \ll \mathbf{0}, q_{i}(x)>0$ and $p(x) \gg \mathbf{0}$. Notice that, although taking distinct index goods $i$ and $j$ in the above system leads to distinct vectors $\left(q_{i}(x), q_{-i}(x)\right)$ and $\left(q_{j}(x), q_{-j}(x)\right)$, the preference gradient, $p(x)$, is invariant with respect to the choice of the index good. Moreover, the fact that $q_{i}(x)=p_{i}(x)$ for the index good $i$ is due to the normalization of income to one.

The non-trivial part in the proof of Proposition 2 is to show that $\partial l_{i}\left(x_{-i} \mid x\right)$ is a singleton if and only if $\succsim$ is differentiable at $x$. Although the argument for the "only if" direction is technical, we provide some intuition in our proof (see Sections 3.2-3.3). For the "if" direction on the other hand, the concept of an ordient that was introduced in Renou and Schlag (2014) may be helpful towards conveying some geometric intuition. Recall first that for $z, x \in X$ with $z \neq x, z-x$ is an improvement [resp. worsening] direction at $x$ if there exists $\lambda^{*}>0$ such that $x+\lambda(z-x) \succ x$ [resp. $x \succ x+\lambda(z-x)$ ] for all $\lambda \in$ $\left(0, \lambda^{*}\right)$ with $x+\lambda(z-x) \in X$. Considering now the plane $H_{p, x}:=\{z \in X: p z=p x\}$ and the interior half-planes $H_{p, x}^{+}:=\{z \in X: p z>p x\}$ and $H_{p, x}^{-}:=\{z \in X: p z<p x\}$, we say that $p \in \mathbb{R}^{n} \backslash\{\mathbf{0}\}$ is an increasing [resp. decreasing] ordient at $x$ if $z-x$ is an improvement [resp. worsening] direction at $x$ for any $z \in H_{p, x}^{+}$[resp. $z \in H_{p, x}^{-}$]. Obviously, $p$ is a preference gradient at $x$ only if it is an increasing ordient at $x$. Moreover, as we establish in the Online Appendix, $p$ supports $\mathcal{U}_{x}$ at $x$ only if it is a decreasing ordient at $x$. For convex preferences, finally, $p$ supports $\mathcal{U}_{x}$ at $x$ if and only if it is a decreasing ordient at $x$.

If $p$ is both an increasing and decreasing ordient at $x$, then it will be referred to as an ordient at $x$. Intuitively, if $p$ is an ordient at $x$, then $\left\{H_{p, x}^{-}, H_{p, x}^{+}\right\}$partitions $X \backslash H_{p, x}$ into improvement and worsening directions: $z-x$ with $z \in X \backslash H_{p, x}$ is an improvement [resp. worsening] direction at $x$ if and only if $z \in H_{p, x}^{+}$[resp. $z \in H_{p, x}^{-}$]. Restricting attention hereafter to strictly convex preferences, it is trivial to check that this partitioning means that $H_{p, x}$ uniquely separates the sets $\mathcal{U}_{x}$ and $X \backslash \mathcal{U}_{x}$ locally at $x$; in geometric terms, $H_{p, x}$ is tangential to $\mathcal{I}_{x}$ at $x$ if $p$ is an ordient at $x$. Moreover, $p$ is a preference gradient at $x$ only if it supports $\mathcal{U}_{x}$ at $x$ properly -thus, only if it is an ordient at $x$. Furthermore, $\succsim$ is differentiable at $x$ only if the collection of vectors that support $\mathcal{U}_{x}$ at $x$ properly is at most a singleton. Hence, $\succsim$ is differentiable at $x$ if and only if there exists a unique preference gradient at $x$. Consider now an arbitrary consumption bundle $x \in X$ and let $p, p^{\prime} \in \mathbb{R}_{++}^{n}$ be such that $x(p)=x=x\left(p^{\prime}\right)$. As both $p$ and $p^{\prime}$ support $\mathcal{U}_{x}$ at $x$, the fact that $\succsim$ is strictly convex implies that both support $\mathcal{U}_{x}$ at $x$ properly. If $\succsim$ is differentiable at $x$, therefore, we must have $p=p^{\prime}$, while the hyperplane $H_{p, x}$ must be tangential to the indifference curve $\mathcal{I}_{x}$ at $x$.

We move on to analysing invertible demand functions that are, in addition, continuous. Recall that, following Neilson (1991), we say that a weak order $\succsim$ on $X$ is weakly $C^{1}$ or weakly smooth if, for all $x \in X$, $\mathcal{I}_{x}$ is a $C^{1}$ manifold of dimension $n-1$.

Proposition 3 Suppose that the onto demand function $x: Y \rightarrow X$ for some $Y \subseteq \mathbb{R}_{++}^{n}$ is generated by the continuous weak order $\succsim$ on $X$. The following are equivalent.

(i). $\succsim$ is differentiable.

(ii). $Y$ is open and $X(\cdot)$ is injective and continuous (thus, a homeomorphism).

(iii). $\succsim$ is weakly smooth.

Therefore, under strict convexity and strict monotonicity, the notions of preference differentiability (Rubinstein, 2006) and weak smoothness (Neilson, 1991) are equivalent and fundamentally related to the continuity of both the direct and inverse demand functions generated by these preferences. 


\subsection{Examples}

We illustrate our main result with two examples. The first presents a strictly convex, strictly monotonic and continuous preference relation that is differentiable but non-smooth. As such, it shows that our characterization does indeed expand the class of preference relations that were hitherto known to generate invertible demand functions. The second example presents a strictly convex, strictly monotonic, continuous and even homothetic preference relation that is non-differentiable, and hence not even weakly smooth. In conjunction with Proposition 4 that is stated in the next subsection, this example clarifies that preference differentiability may fail even in the presence of a rich structure.

Example 1: strictly convex, strictly monotonic and differentiable but non-smooth preferences (Figure 1).

Consider the weak order $\succsim$ on $X=(2 e,+\infty) \times(0,1)$ that is represented by the utility function

$$
u(x):= \begin{cases}\ln x_{1}+\ln x_{2}, & \text { if } x_{1} x_{2} \leq e \\ \frac{\ln x_{1}}{1-\ln x_{2}}, & \text { otherwise }\end{cases}
$$

It is easy to verify that $\succsim$ is continuous, strictly increasing and strictly convex on $X$. Let $S:=\{x \in$ $\left.X: x_{1} x_{2} \leq e\right\}$. For $\bar{u} \in \mathbb{R}$, the indifference set $\{x \in X: u(x)=\bar{u}\}$ coincides with the graph of the function $x_{2}: \mathbb{R}_{++} \rightarrow \mathbb{R}_{++}$that is implicitly defined by $x_{2}:=e^{\bar{u}} / x_{1}$ if $x \in S$ and $x_{2}:=e x_{1}^{-(1 / \bar{u})}$ if $x \in X \backslash S$. Therefore, for any $\bar{x} \in X$ we have $l_{2}\left(x_{1} \mid \bar{x}\right)=e^{u(\bar{x})} / x_{1}$ if $\bar{x} \in S$ and $l_{2}\left(x_{1} \mid \bar{x}\right)=e x_{1}^{-(1 / u(\bar{x}))}$ if $\bar{x} \in X \backslash S$. Moreover, for any $x \in \mathcal{I}_{\bar{x}}$, we get $l_{2}^{\prime}\left(x_{1} \mid \bar{x}\right)=-l_{2}\left(x_{1} \mid \bar{x}\right) / x_{1}=-x_{2} / x_{1}$ on $S$ and $l_{2}^{\prime}\left(x_{1} \mid \bar{x}\right)=$ $-u(\bar{x})^{-1} l_{2}\left(x_{1} \mid \bar{x}\right) / x_{1}=-u(\bar{x})^{-1} x_{2} / x_{1}$ on $X \backslash S$. In particular, since $u(\bar{x})=1$ when $\bar{x}_{1} \bar{x}_{2}=e$, it follows that $\succsim$ is also differentiable on $X$ and, by equations (3) - (5), the gradient of its indifference-projection function, $q(x)$, and the preference gradient and inverse demand, $p(x)$, at $x$ are given by

$$
q(x)= \begin{cases}\left(-\frac{x_{2}}{x_{1}}, \frac{1}{2 x_{2}}\right), & \text { if } x_{1} x_{2} \leq e \\ \left.-\frac{x_{2}\left(1-\ln x_{2}\right)}{x_{1} \ln x_{1}}, \frac{\ln x_{1}}{x_{2}\left(1+\ln \left(x_{1} / x_{2}\right)\right)}\right), & \text { otherwise }\end{cases}
$$

and

$$
p(x)= \begin{cases}\left(\frac{1}{2 x_{1}}, \frac{1}{2 x_{2}}\right), & \text { if } x_{1} x_{2} \leq e \\ \left(\frac{1-\ln x_{2}}{x_{1}\left(1+\ln \left(x_{1} / x_{2}\right)\right)}, \frac{\ln x_{1}}{x_{2}\left(1+\ln \left(x_{1} / x_{2}\right)\right)}\right), & \text { otherwise }\end{cases}
$$

Notice, however, that $u(\cdot)$ is not even partially differentiable when $x_{1} x_{2}=e$, while the gradient of $u(\cdot)$ elsewhere is given by

$$
\nabla u(x)= \begin{cases}\left(\frac{1}{x_{1}}, \frac{1}{x_{2}}\right), & \text { if } x_{1} x_{2}<e \\ \left(\frac{1}{x_{1}\left(1-\ln x_{2}\right)}, \frac{\ln x_{1}}{x_{2}\left(1-\ln x_{2}\right)^{2}}\right), & \text { if } x_{1} x_{2}>e\end{cases}
$$

In fact, note that no utility function that represents $\succsim$ can be differentiable on $S_{0}:=\left\{x \in X: x_{1} x_{2}=e\right\}$. To see this, let $f(u(\cdot))$ be such a utility function, where $f: \mathbb{R} \rightarrow \mathbb{R}$ is a strictly increasing and continuously differentiable transformation. Letting now a sequence $\left(x^{n}\right)$ in $X$ converge to $x \in S_{0}$, we have 
$f^{\prime}\left(u\left(x^{n}\right)\right) \nabla u\left(x^{n}\right) \quad$ converging to $\lim _{z \nearrow 1} f^{\prime}(z)\left(\frac{1}{x_{1}}, \frac{1}{x_{2}}\right) \quad$ from within $S$, and to $\lim _{z \searrow 1} f^{\prime}(z)\left(\frac{1}{x_{1}} \cdot \frac{1}{1-\ln x_{2}}, \frac{1}{x_{2}} \cdot \frac{\ln x_{1}}{\left(1-\ln x_{2}\right)^{2}}\right)$ from outside of $S$. But these two limits are distinct.

Example 2: strictly convex, strictly monotonic, homothetic and non-differentiable preferences (Figure 2).

Consider now the weak order $\succsim$ on $X=\mathbb{R}_{++}^{2}$ that is represented by the utility function

$$
u(x):= \begin{cases}x_{1}^{\frac{2}{3}} x_{2}^{\frac{1}{3}}, & \text { if } x_{1} \leq x_{2} \\ x_{1}^{\frac{1}{3}} x_{2}^{\frac{2}{3}}, & \text { otherwise }\end{cases}
$$

It is again easily verifiable that this $\succsim$ is continuous, strictly increasing, strictly convex and also homothetic on $X$. But $\succsim$ is not differentiable anywhere on $S_{0}:=\left\{x \in X: x_{1}=x_{2}\right\}$, and neither $u(\cdot)$ nor any other utility function that also represents $\succsim$ is differentiable on this set. ${ }^{5}$

Figure 1: Strictly convex, strictly monotonic and differentiable but non-smooth preferences (Example 1)

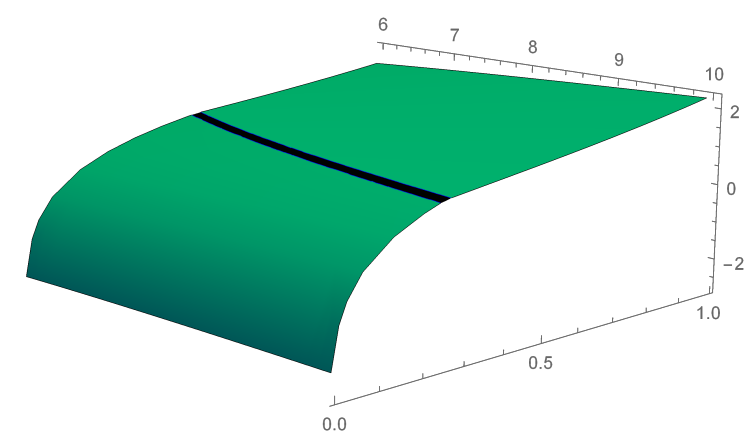

(a) The utility function is kinked at $x_{1} \cdot x_{2}=e$

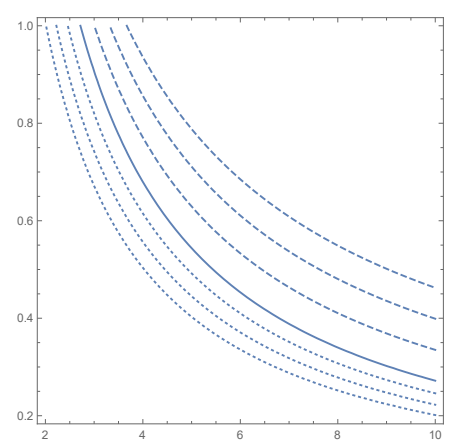

(b) The indifference curves are smooth (solid for $\bar{u}=1$ )

Figure 2: Strictly convex, strictly monotonic, homothetic and non-differentiable preferences (Example 2)

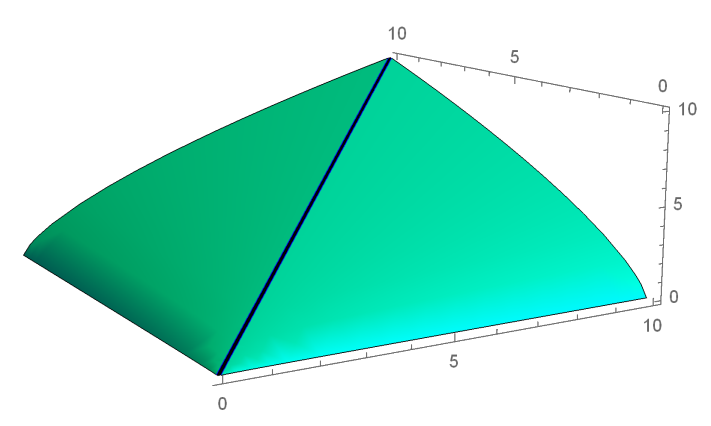

(a) The utility function is kinked at $x_{1}=x_{2}$

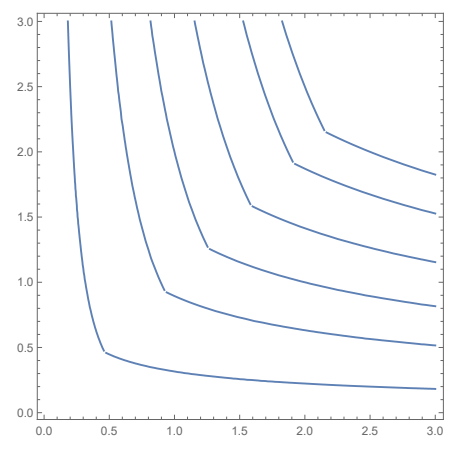

(b) The indifference curves are kinked at $x_{1}=x_{2}$

\subsection{The Special Case of Quasilinear or Homothetic Preferences}

Recall that a preference relation $\succsim$ on $X$ is homothetic if, for all $x, y \in X$ and $\lambda>0, x \succsim y$ implies $\lambda x \succsim \lambda y$. A homothetic preference relation is representable by a utility function $u(\cdot)$ that is homogeneous of degree

\footnotetext{
${ }^{5}$ For another such example with a more complicated functional form, the reader is referred to Hurwicz and Uzawa (1971).
} 
1 , hence satisfying $u(\lambda x)=\lambda u(x)$ for all $x \in X$ and $\lambda>0$. Denoting by $\mathbf{e}_{i} \in \mathbb{R}_{+}^{n}$ the vector defined by $\mathbf{e}_{i}^{i}=1$ and $\mathbf{e}_{i}^{j}=0$ for $j \neq i$, recall next that $\succsim$ is quasilinear with respect to good $i$ if $x \succsim y$ implies $x+\lambda \mathbf{e}_{i} \succsim y+\lambda \mathbf{e}_{i}$ and $x+\lambda \mathbf{e}_{i} \succ x$ for all $\lambda>0$ and $x \in X$ (Mas-Colell, Whinston and Green, 1995). A preference relation that is quasilinear with respect to good $i$ is representable by a utility function $u(\cdot)$ with the property that $u(x):=x_{i}+v\left(x_{-i}\right)$ for some function $v: X_{-i} \rightarrow \mathbb{R}$.

The next result clarifies that, within the class of preference relations that satisfy the conditions of Theorem 1 and are also homothetic or quasilinear, representability of a relation in this class by a continuously differentiable utility function is equivalent to the differentiability or weak smoothness of that relation.

Proposition 4 The following are equivalent for a strictly convex, strictly monotonic and continuous weak order $\succsim$ on $X$ that is quasilinear or homothetic:

(i). $\succsim$ is differentiable.

(ii). $\succsim$ is weakly smooth.

(iii). $\succsim$ is representable by a continuously differentiable utility function.

Example 2 above provides an illustration of this result by presenting preferences that are strictly convex, strictly monotonic and even homothetic but fail differentiability and hence do not admit a smooth utility representation.

\subsection{The Law of Demand}

As mentioned in the introduction, invertibility is a necessary condition for any demand function $x$ : $Y \rightarrow X$ that satisfies the "strict law of demand". This is formally defined by the condition that, for all prices $p, p^{\prime} \in Y$,

$$
\left(x(p)-x\left(p^{\prime}\right)\right) \cdot\left(p-p^{\prime}\right)<0 .
$$

A complete, transitive and continuous preference relation that is also homothetic is well-known to generate a demand function that satisfies the "weak law of demand" where the above inequality is not necessarily strict (Hildenbrand, 1994). Mitjuschin and Polterovich (1978) (see also Kannai (1989) for an extension) provided a different sufficient condition for a $C^{1}$ demand function that is derived from a $C^{2}$ and strictly increasing utility function $u(\cdot)$ to satisfy the strict version of the law. In addition to double smoothness, that condition requires concavity of $u(\cdot)$ and also that

$$
-\frac{z \cdot \nabla^{2} u(z) \cdot z}{z \cdot \nabla u(z)}<4
$$

be satisfied for all $z \in \mathbb{R}_{++}^{n}$. This condition is not easily interpretable behaviourally. In addition, since concavity and smoothness of a given utility representation are not ordinal properties, the condition itself is not ordinal. Despite the existence of this sufficient condition, however, little is known about necessary conditions on the preferences that generate demand functions that satisfy this law. A novel implication of our analysis (Propositions 1-2) that makes a contribution in this direction can be stated as follows.

Corollary 2.1 A rational and onto demand function satisfies the strict law of demand only if it is generated by a strictly convex, strictly monotonic and differentiable or weakly smooth preference relation. 
While the onto-demand requirement is somewhat restrictive and one hopes that it will be relaxed in future work, it allows for uncovering what appear to be the first behaviourally interpretable necessary conditions on preferences for a class of demand functions that satisfy this law. We refer the reader to Aguiar, Hjertstrand, and Serrano (2020) for an independent recent study that contributes further to the uncovering of the behavioural origins of the law of demand by analysing the case of finite data that are compatible with demand functions satisfying the weak version of the law.

\section{Proofs}

Recall that, for $x \in A \subseteq X$ and $\varepsilon>0, \mathcal{B}_{\varepsilon}(x)$ denotes the open ball in $\mathbb{R}^{n}$ with center $x$ and radius $\varepsilon$. In what follows, we denote also by $\|x\|$ the Euclidean norm of $x$, and define the index sets $\mathcal{N}:=\{1, \ldots, n\}$ as well as

$$
\mathcal{N}_{x}^{+}:=\left\{i \in \mathcal{N}: x_{i}>0\right\}, \quad \mathcal{N}_{x}^{-}:=\left\{i \in \mathcal{N}: x_{i}<0\right\} .
$$

Moreover, $\operatorname{int}(A), \mathrm{bd}(A)$ and $\mathrm{cl}(A)$ denote, respectively, the interior, boundary and closure of $A$. Finally, taking $i \in \mathcal{N}$, we let $x_{i}$ and $A^{i}$ denote, respectively, the projections of $x$ and $A$ on $\mathbb{R}$ - the $i$ th dimension of $\mathbb{R}^{n}$. By contrast, $x_{-i}$ and $A^{-i}$ will denote, respectively, the projections of $x$ and $A$ on $\mathbb{R}^{n-1}$ - the resulting subspace when the $i$ th dimension is removed from $\mathbb{R}^{n}$. Taking also $j \in \mathcal{N} \backslash\{i\}$, we will have $x_{-(i, j)}$ denote the projection of $x$ on $\mathbb{R}^{n-2}$ - the resulting subspace when both the $i$ th and $j$ th dimensions are removed from $\mathbb{R}^{n}$.

\subsection{Proof of Proposition 1}

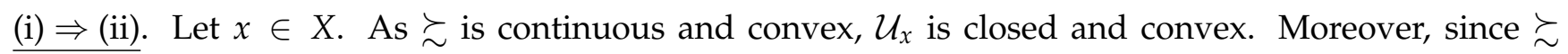
is strictly monotonic, it must be $x \in \operatorname{bd}\left(\mathcal{U}_{x}\right)$ while int $\left(\mathcal{U}_{x}\right) \neq \varnothing$. To check the first claim, suppose to the contrary that $x \in \operatorname{int}\left(\mathcal{U}_{x}\right)$. We have then $\left(x_{i}-\varepsilon / 2, x_{-i}\right) \in \mathcal{B}_{\mathcal{\varepsilon}}(x) \subset \mathcal{U}_{x}$ for some $\varepsilon>0$, and a contradiction obtains because strict monotonicity necessitates that $x \succ\left(x_{i}-\varepsilon / 2, x_{-i}\right)$. For the second claim, notice that, $X$ being open, we have $\mathcal{B}_{\delta}(x) \subset X$ for some $\delta>0$. Letting then $x^{\prime} \in \mathcal{B}_{\delta}(x)$ be given by $x_{i}^{\prime}=x_{i}+\delta / 2$ for $i \in \mathcal{N}$, strict monotonicity ensures that $x^{\prime} \succ x$; thus, $\mathcal{B}_{\delta / 4}\left(x^{\prime}\right) \subset \mathcal{U}_{x}$.

Given the observations above, it follows from the Supporting Hyperplane Theorem - see for instance Lemma 7.7 in Aliprantis and Border (2006) - that some $p \in \mathbb{R}^{n} \backslash\{\mathbf{0}\}$ supports $\mathcal{U}_{x}$ at $x$. In fact, by the following two lemmas, it must be $p \in \mathbb{R}_{++}^{n}$ while $x \in \max _{\succsim}\{z \in X: p z \leq p x\}$.

Lemma 3.1 Let $\succsim$ be a continuous weak order on $X$. For any $x \in X, p \in \mathbb{R}^{n} \backslash\{\mathbf{0}\}$ supports $\mathcal{U}_{x}$ at $x$ only if $x \in \max _{\succsim}\{z \in X: p z \leq p x\}$.

Proof. Let $p \in \mathbb{R}^{n} \backslash\{\boldsymbol{0}\}$ support $\mathcal{U}_{x}$ at $x$. We need to show that $z \in X \backslash\{x\}$ and $p z \leq p x$ implies $x \succsim z$. As this is obvious when $p z<p x$, suppose that $p z=p x$ and assume to the contrary that $z \succ x$; i.e., that $z \in \mathcal{U}_{x} \backslash \mathcal{I}_{x}$. Since $\succsim$ is continuous, the latter set is open; thus, $\mathcal{B}_{\varepsilon}(z) \subset \mathcal{U}_{x} \backslash \mathcal{I}_{x}$ for sufficiently small $\varepsilon>0$. Take now $z^{\prime} \in \mathcal{B}_{\varepsilon}(z)$ given by $z_{i}^{\prime}:=z_{i}+\frac{\varepsilon}{2}$ if $p_{i} \leq 0$ and $z_{i}^{\prime}:=z_{i}-\frac{\varepsilon}{2}$ if $p_{i}>0$, for $i \in \mathcal{N}$. Since $p \neq \mathbf{0}$, we have $p z^{\prime}<p z=p x$, which contradicts, however, that $p$ supports $\mathcal{U}_{x}$ at $x$.

Lemma 3.2 Let $\succsim$ be a strictly monotonic and continuous weak order on $X$. For any $x \in X, p \in \mathbb{R}^{n} \backslash\{\mathbf{0}\}$ supports $\mathcal{U}_{x}$ at $x$ only if $p \in \mathbb{R}_{++}^{n}$. 
Proof. Let $(p, x) \in \mathbb{R}^{n} \backslash\{\mathbf{0}\} \times X$ be such that $p$ supports $\mathcal{U}_{x}$ at $x$. To argue ad absurdum, suppose that $p_{i} \leq 0$ for some $i \in \mathcal{N}$. $X$ being open, we have $\mathcal{B}_{\delta}(x) \subset X$ for some $\delta>0$. Taking then $z \in \mathcal{B}_{\delta}(x)$ such that $z_{i}>x_{i}$ and $z_{j}=x_{j}$ for $j \in \mathcal{N} \backslash\{i\}$, we get $p z \leq p x$. As $\succsim$ is strictly monotonic, however, we also have $z \succ x$. Lemma 3.1 gives the desired contradiction.

There exists thus $p \in \mathbb{R}_{++}^{n}$ with $x \in \max _{\succsim}\{z \in X: p z \leq p x\}$. Equivalently (since $x \in X \subseteq \mathbb{R}_{++}^{n}$ ), there exists $\widetilde{p}:=p / p x \in \mathbb{R}_{++}^{n}$ such that $x \in \max _{\succsim}\{z \in X: \widetilde{p} z \leq 1\}$. Defining therefore $Y$ as in the main text, the mapping $x: Y \rightarrow \mathbb{R}_{++}^{n}$ given by $x(p):=\max _{\succsim}\{z \in X: p z \leq 1\}$ is an onto demand correspondence. That it is also single-valued follows from the strict convexity of $\succsim$.

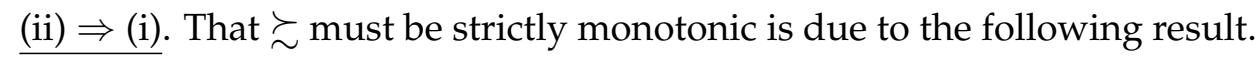

Lemma 3.3 Let $\succsim$ be a weak order on $X$. The onto demand function $x: Y \rightarrow X\left(Y \subseteq \mathbb{R}_{++}^{n}\right)$ is generated by $\succsim$ only if the latter is strictly monotonic on $X$.

Proof. Let $x, z \in X$ be such that $x \geq z$ with $x \neq z$. Since $x(\cdot)$ is onto, single-valued and generated by $\succsim$, there exists $p \in \mathbb{R}_{++}^{n}$ such that $x=x(p)$. Hence, $p x \leq 1$ while $x \succ z^{\prime}$ for all $z^{\prime} \in X \backslash\{x\}$ with $p z^{\prime} \leq 1$. Notice now that $x \neq z, z-x \leq \mathbf{0}$ and $p \gg \mathbf{0}$ together imply that $p(z-x)<0$. We have therefore $p z<p x \leq 1$ and thus $x \succ z$.

Given now the strict monotonicity and continuity of $\succsim$ and the single-valuedness of $x(\cdot)$, that $\succsim$ must be also strictly convex follows from the equivalence result in Bilancini and Boncinelli (2010).

\subsection{Supporting Results for the Proof of Proposition 2}

Lemma 3.4 Let the (onto) demand function $x: Y \rightarrow X$ for some $Y \subseteq \mathbb{R}_{++}^{n}$ be generated by the strictly convex and strictly monotonic continuous weak order $\succsim$ on $X$. For any $(p, x) \in \mathbb{R}_{++}^{n} \times X$, the following are equivalent.

(i) $p$ supports $\mathcal{I}_{x}$ at $x$ locally (i.e., there exists $\varepsilon>0$ such that $p x \leq p z$ for any $z \in \mathcal{B}_{\varepsilon}(x) \cap \mathcal{I}_{x}$ ).

(ii) $p$ supports $\mathcal{U}_{x}$ at $x$ properly.

(iii). $x=x(p)$.

Proof. (ii) $\Rightarrow$ (i) being trivially true, we will establish first that (i) $\Rightarrow$ (ii). Take thus any $x \in X$ and let $(\varepsilon, p) \in \mathbb{R}_{++} \times \mathbb{R}_{++}^{n}$ be such that $p x \leq p z$ for any $z \in \mathcal{B}_{\varepsilon}(x) \cap \mathcal{I}_{x}$.

We will show first that $p$ supports $\mathcal{B}_{\varepsilon}(x) \cap\left(\mathcal{U}_{x} \backslash \mathcal{I}_{x}\right)$ at $x$ properly. To this end, we need to show that $p x<p z^{\prime}$ for any $z^{\prime} \in \mathcal{B}_{\varepsilon}(x)$ such that $z^{\prime} \succ x$. Take then any such $z^{\prime}$ and let $\epsilon:=z^{\prime}-x$. By the strict monotonicity of $\succsim$, we must have $\mathcal{N}_{\epsilon}^{+} \neq \varnothing$. Notice also that, the claim being obvious if $\mathcal{N}_{\epsilon}^{+}=\mathcal{N}$, we may take $\mathcal{N} \backslash \mathcal{N}_{\epsilon}^{+}$to be non-empty. And as the claim is again obvious if $\epsilon_{i}=0$ for all $i \in \mathcal{N} \backslash \mathcal{N}_{\epsilon}^{+}$, we may take in fact that $\mathcal{N}_{\epsilon}^{-} \neq \varnothing$. Let now $\mu \in[0,1]$ and define $x^{\mu} \in \mathcal{B}_{\varepsilon}(x)$ by $x_{i}^{\mu}:=x_{i}+\mu \epsilon_{i}$ for $i \in \mathcal{N} \backslash \mathcal{N}_{\epsilon}^{-}$and $x_{i}^{\mu}:=x_{i}+\epsilon_{i}$ for $i \in \mathcal{N}_{\epsilon}^{-}$. As $\mathcal{N}_{\epsilon}^{-} \neq \varnothing$ while $x^{1}=z^{\prime}$, we have $x^{1} \succ x \succ x^{0}$ again due to the strict monotonicity of $\succsim$. The relation being also continuous, there exists $\mu_{0} \in(0,1)$ such that $\mu_{0} x^{1}+\left(1-\mu_{0}\right) x^{0} \in \mathcal{B}_{\varepsilon}(x) \cap \mathcal{I}_{x}$. The latter point is given though by $x_{i}+\mu_{0} \epsilon_{i}$ for $i \in \mathcal{N} \backslash \mathcal{N}_{\epsilon}^{-}$and $x_{i}+\epsilon_{i}$ 
for $i \in \mathcal{N}_{\epsilon}^{-}$. That is, $\mu_{0} x^{1}+\left(1-\mu_{0}\right) x^{0}=x^{\mu_{0}}$ and the claim now follows since

$$
\begin{aligned}
p x \leq p x^{\mu_{0}} & =\sum_{i \in \mathcal{N} \backslash \mathcal{N}_{\epsilon}^{-}} p_{i}\left(x_{i}+\mu_{0} \epsilon_{i}\right)+\sum_{i \in \mathcal{N}_{\epsilon}^{-}} p_{i}\left(x_{i}+\epsilon_{i}\right) \\
& =\sum_{i \in \mathcal{N}_{\epsilon}^{+}} p_{i}\left(x_{i}+\mu_{0} \epsilon_{i}\right)+\sum_{i \in \mathcal{N}_{\epsilon}^{-}} p_{i}\left(x_{i}+\epsilon_{i}\right) \\
& <\sum_{i \in \mathcal{N}_{\epsilon}^{+}} p_{i}\left(x_{i}+\epsilon_{i}\right)+\sum_{i \in \mathcal{N}_{\epsilon}^{-}} p_{i}\left(x_{i}+\epsilon_{i}\right)=\sum_{i \in \mathcal{N}} p_{i}\left(x_{i}+\epsilon_{i}\right)=p(x+\epsilon)=p z^{\prime}
\end{aligned}
$$

where the strict inequality is because $p \in \mathbb{R}_{++}^{n}$ and $\mathcal{N}_{\epsilon}^{+} \neq \varnothing$ while the second and third equalities use the fact that $\mathcal{N} \backslash \mathcal{N}_{\epsilon}^{-}=\mathcal{N}_{\epsilon}^{+} \cup\left\{i \in \mathcal{N}: \epsilon_{i}=0\right\}$.

Next we will show that $p$ supports also $\mathcal{B}_{\varepsilon}(x) \cap \mathcal{I}_{x}$ at $x$ properly. To argue by contradiction, suppose that there exists $z^{\prime \prime} \in \mathcal{B}_{\varepsilon}(x) \cap \mathcal{I}_{x}$ for which $p x=p z^{\prime \prime}$. For any $\lambda \in(0,1)$ then we have that $\lambda z^{\prime \prime}+(1-\lambda) x \succ$ $x$, by the strict convexity of $\succsim$, while $p\left(\lambda z^{\prime \prime}+(1-\lambda) x\right)=p x$. Taking though $\lambda<\varepsilon /\left\|x-z^{\prime \prime}\right\|$ ensures that $\lambda z^{\prime \prime}+(1-\lambda) x \in \mathcal{B}_{\varepsilon}(x)$. Which contradicts that $p$ supports $\mathcal{B}_{\varepsilon}(x) \cap\left(\mathcal{U}_{x} \backslash \mathcal{I}_{x}\right)$ at $x$ properly.

We have therefore established that $p$ supports $\mathcal{B}_{\varepsilon}(x) \cap \mathcal{U}_{x}$ at $x$ properly. But then $p$ must in fact support $\mathcal{U}_{x}$ at $x$ properly. For otherwise, if there existed $\widetilde{z} \in \mathcal{U}_{x}$ with $p \widetilde{z} \leq p x$, then for any $\lambda \in(0,1)$ we would have $\lambda \widetilde{z}+(1-\lambda) x \succ x$ while $p(\lambda \widetilde{z}+(1-\lambda) x) \leq p x$. A contradiction would obtain then because taking $\lambda<\varepsilon /\|x-\widetilde{z}\|$ ensures that $\lambda \widetilde{z}+(1-\lambda) x \in \mathcal{B}_{\varepsilon}(x)$.

To complete the proof, observe that (ii) $\Rightarrow$ (iii) is an immediate implication of Lemmas 3.1-3.2. To establish the contrapositive of (iii) $\Rightarrow$ (ii), suppose that $p$ does not support $\mathcal{U}_{x}$ at $x$ properly. As it cannnot support $\mathcal{I}_{x}$ at $x$ locally either (recall the contrapositive of (i) $\Rightarrow$ (ii)), there must exist $\varepsilon>0$ and $z \in\left(\mathcal{B}_{\varepsilon}(x) \cap \mathcal{I}_{x}\right) \backslash\{x\}$ with $p z<p x$. But then it cannot be that $x=\max _{\succsim}\{z \in X: p z \leq p x\}=x(p)$.

Lemma 3.5 Let $\succsim$ be a strictly convex and strictly monotonic, continuous weak order on $X$. For any $(x, z, i) \in$ $X \times \mathcal{I}_{x} \times \mathcal{N}$, the mapping $l_{i}(\cdot \mid x): \mathcal{I}_{x}^{-i} \rightarrow \mathcal{I}_{x}^{i}$ is a locally strictly convex function with $\partial l_{i}\left(z_{-i} \mid x\right) \subseteq \mathbb{R}_{--}^{n-1}$.

Proof. $\succsim$ being a strictly convex and strictly monotonic continuous weak order, it can be represented by a continuous, strictly monotonic and strictly quasi-concave utility function $u: X \rightarrow \mathbb{R}$. Consider then an arbitrary $(i, x) \in \mathcal{N} \times X$. To see that $l_{i}(\cdot \mid x)$ is a function, take any $z \in \mathcal{I}_{x}$ and observe that we cannot have $z_{i}, z_{i}^{\prime} \in l_{i}\left(z_{-i} \mid x\right)$ with $z_{i}>z_{i}^{\prime}$. For this would imply that $z \sim x \sim\left(z_{i}^{\prime}, z_{-i}\right)$ which is absurd under strict monotonicity.

We will establish next that $\mathcal{I}_{x}^{-i}$ is locally convex at $z_{-i}$. To this end, let $\varepsilon_{0}>0$ be such that $\mathcal{B}_{\varepsilon_{0}}(z) \subset X$. Let also $\Delta:=u(x)-u\left(z_{i}-\varepsilon_{0} / 2, z_{-i}\right)=u(z)-u\left(z_{i}-\varepsilon_{0} / 2, z_{-i}\right)>0$, the inequality due to $u(\cdot)$ being strictly monotonic. The utility function being also continuous, taking a sufficiently small $\varepsilon_{1}>0$ ensures that $\left|u\left(z_{i}-\varepsilon_{0} / 2, \cdot\right)-u\left(z_{i}-\varepsilon_{0} / 2, z_{-i}\right)\right|<\Delta$ on $\mathcal{B}_{\varepsilon_{1}}\left(z_{-i}\right) \subset \mathcal{B}_{\varepsilon_{0}}(z)_{-i}$. And as

$$
\begin{aligned}
\left\|\lambda v_{-i}+(1-\lambda) y_{-i}-z_{-i}\right\| & =\left\|\lambda\left(v_{-i}-z_{-i}\right)+(1-\lambda)\left(y_{-i}-z_{-i}\right)\right\| \\
& \leq \lambda\left\|v_{-i}-z_{-i}\right\|+(1-\lambda)\left\|y_{-i}-z_{-i}\right\|<\lambda \varepsilon_{1}+(1-\lambda) \varepsilon_{1}=\varepsilon_{1}
\end{aligned}
$$

we get that

$$
u\left(z_{i}-\varepsilon_{0} / 2, \lambda v_{-i}+(1-\lambda) y_{-i}\right)<u(z)=u(x)
$$

for any $v_{-i}, y_{-i} \in \mathcal{B}_{\varepsilon_{1}}\left(z_{-i}\right) \cap \mathcal{I}_{x}^{-i}$ and any $\lambda \in(0,1)$. Take then any $v, y \in \mathcal{I}_{x} \cap \mathcal{B}_{\varepsilon_{1}}(z)$ and any $\lambda \in(0,1)$. Since $u(v)=u(x)=u(y)$, the strict quasi-concavity of $u(\cdot)$ gives

$$
u(x)<u(\lambda v+(1-\lambda) y)=u\left(\lambda v_{i}+(1-\lambda) y_{i}, \lambda v_{-i}+(1-\lambda) y_{-i}\right)
$$


Consider now the $[0,1] \rightarrow \mathbb{R}_{++}$function $z_{i}(\mu):=\mu\left(z_{i}-\varepsilon_{0} / 2\right)+(1-\mu)\left(\lambda v_{i}+(1-\lambda) y_{i}\right)$. This gives $\left(z_{i}(\mu), \lambda v_{-i}+(1-\lambda) y_{-i}\right) \in X$ for any $\mu \in[0,1]$. Moreover, in light of (6)-(7), the intermediate-value theorem ensures that

$$
\exists \mu_{0} \in(0,1): u\left(z_{i}\left(\mu_{0}\right), \lambda v_{-i}+(1-\lambda) y_{-i}\right)=u(x)
$$

and thus $\lambda z_{-i}+(1-\lambda) y_{-i} \in \mathcal{I}_{x}^{-i}$, as required.

Observe next that, by the strict monotonicity of $u(\cdot),(7)-(8)$ require that $z_{i}\left(\mu_{0}\right)<\lambda v_{i}+(1-\lambda) y_{i}$; equivalently, that

$$
l_{i}\left(\lambda v_{-i}+(1-\lambda) y_{-i} \mid x\right)<\lambda l_{i}\left(v_{-i} \mid x\right)+(1-\lambda) l_{i}\left(y_{-i} \mid x\right)
$$

Hence, $l_{i}(\cdot \mid x)$ is strictly convex on $\mathcal{B}_{\varepsilon_{1}}\left(x_{-i}\right) \cap \mathcal{I}_{x}^{-i}$.

To see finally that the local subgradients of $l_{i}(\cdot \mid x)$ at $z_{-i}$ all lie in $\mathbb{R}_{--}^{n-1}$, let $q \in \mathbb{R}^{n-1}$ be a local subgradient. We have

$$
l_{i}\left(y_{-i} \mid x\right)-l_{i}\left(z_{-i} \mid x\right) \geq \sum_{j \in \mathcal{N} \backslash\{i\}} q_{j}\left(y_{j}-z_{j}\right) \quad \forall y_{-i} \in \mathcal{B}_{\varepsilon_{1}}\left(x_{-i}\right) \cap \mathcal{I}_{x}^{-i}
$$

To argue ad absurdum, suppose that $q_{k} \geq 0$ for some $k \in \mathcal{N} \backslash\{i\}$. Define also the function $[0,1] \rightarrow \mathbb{R}_{++}^{n}$ by $z_{i}(\widetilde{\mu}):=z_{i}-(1-\widetilde{\mu}) \varepsilon_{1} / 2, z_{k}(\widetilde{\mu}):=z_{k}+\widetilde{\mu} \varepsilon_{1} / 2$, and $z_{j}(\widetilde{\mu}):=z_{j}$ for $j \in \mathcal{N} \backslash\{i, k\}$. Obviously, any $\widetilde{\mu} \in[0,1]$ gives $z(\widetilde{\mu}) \in \mathcal{B}_{\varepsilon_{1}}(x)$. And since $u(z(0))<u(z)<u(z(1))$ due to strict monotonicity, the intermediate-value theorem ensures the existence of $z\left(\widetilde{\mu}_{0}\right) \in \mathcal{B}_{\varepsilon_{1}}(z) \cap \mathcal{I}_{x}$. But then (10) gives

$$
\left(1-\widetilde{\mu}_{0}\right) \varepsilon_{1} / 2=z_{i}-z_{i}\left(\widetilde{\mu}_{0}\right)=l_{i}\left(z_{-i} \mid x\right)-l_{i}\left(z_{-i}\left(\widetilde{\mu}_{0}\right) \mid x\right) \leq q_{-i}\left(z_{-i}-z_{-i}\left(\widetilde{\mu}_{0}\right)\right)=-\widetilde{\mu}_{0} q_{k} \varepsilon_{1} / 2 \leq 0
$$

a contradiction.

Lemma 3.6 Let the (onto) demand function $x: Y \rightarrow X\left(Y \subseteq \mathbb{R}_{++}^{n}\right)$ be generated by the strictly convex and strictly monotonic continuous weak order $\succsim$ on $X$. For any $(x, z, i) \in X \times \mathcal{I}_{x} \times \mathcal{N}$ there exists a bijection $p_{z}: \partial l_{i}\left(z_{-i} \mid x\right) \rightarrow\{p \in Y: z=x(p)\}$ given by

$$
p_{z}\left(q_{-i}\right):=\left(1,-q_{-i}\right) /\left(z_{i}-q_{-i} z_{-i}\right)
$$

Proof. We will establish first that the mapping in question is a function. To this end, take any $(x, z) \in$ $X \times \mathcal{I}_{x}$ and recall that, for any $i \in \mathcal{N}, l_{i}(\cdot \mid x)$ is a locally convex function (Lemma 3.5); thus, the set $\partial l_{i}\left(z_{-i} \mid x\right)$, the local subdifferential of $l_{i}(\cdot \mid x)$ at $z_{-i}$, is non-empty. Letting then $q_{-i} \in \mathbb{R}_{--}^{n-1}$ be a local subgradient of $l_{i}(\cdot \mid x)$ at $z_{-i}$, we can define (uniquely) the quantities

$$
\begin{aligned}
q_{i} & :=1 /\left(z_{i}-q_{-i} z_{-i}\right) \in \mathbb{R}_{++} \\
p & :=q_{i}\left(1,-q_{-i}\right) \in \mathbb{R}_{++}^{n}
\end{aligned}
$$

There exists then $\varepsilon>0$ such that, for any $y \in \mathcal{B}_{\varepsilon}(z) \cap \mathcal{I}_{x}$, we have

$$
\begin{aligned}
p_{i}\left(y_{i}-z_{i}\right)=q_{i}\left(y_{i}-z_{i}\right) & =q_{i}\left(l_{i}\left(y_{-i} \mid x\right)-l_{i}\left(z_{-i} \mid x\right)\right) \\
& \geq q_{i} q_{-i}\left(y_{-i}-z_{-i}\right)=p_{-i}\left(z_{-i}-y_{-i}\right)
\end{aligned}
$$


Clearly, $p$ supports $\mathcal{I}_{x}$ at $z$ locally and, thus, it must be that $z=x(p)$ (Lemma 3.4).

To show next that the function in question is onto, take any $p \in\{\widetilde{p} \in Y: z=x(\widetilde{p})\}$. Since $z=x(p), p$ supports $\mathcal{U}_{z}$ at $z$ properly (see again Lemma 3.4); hence, any $y \in \mathcal{I}_{x} \backslash\{z\}$ gives $p y>p z$. Equivalently,

$$
l_{i}\left(y_{-i} \mid x\right)=y_{i}>z_{i}-p_{-i}\left(y_{-i}-z_{-i}\right) / p_{i}=l_{i}\left(z_{-i} \mid x\right)-p_{-i}\left(y_{-i}-z_{-i}\right) / p_{i}
$$

and thus $q_{-i}:=-p_{-i} / p_{i} \in \mathbb{R}_{--}^{n}$ is a subgradient of $l_{i}(\cdot \mid x)$ at $z_{-i}$. And since $p z=1$ (by Walras' Law), letting $q_{i}:=p_{i}$ suffices for (11)-(12) above to hold.

To show finally that the function is also injective, suppose that $p$ above is the image of two different local subgradients $q_{-i}, \widetilde{q}_{-i} \in \partial l_{i}\left(z_{-i} \mid x\right)$. By (11)-(12) then we have

$$
\widetilde{q}_{i} z_{i}=1+\widetilde{q}_{i} \widetilde{q}_{-i} z_{-i}=1-p_{-i} z_{-i}=1+q_{i} q_{-i} z_{-i}=q_{i} z_{i}
$$

That is, $q_{i}=\widetilde{q}_{i}$ which implies in turn that $q_{-i}=-p_{-i} / q_{i}=-p_{-i} / \widetilde{q}_{i}=\widetilde{q}_{-i}$.

Lemma 3.7 Let $\succsim$ be a strictly convex weak order on $X$. The collection of preference gradients at $x \in X$ is a subset of the collection of $p \in \mathbb{R}^{n} \backslash\{0\}$ that support $\mathcal{U}_{x}$ at $x$ properly.

Proof. Take an arbitrary $z \in \mathcal{U}_{x}$ and observe that, $\succsim$ being strictly convex, we have that $x+\lambda(z-x)=$ $\lambda z+(1-\lambda) x \succ x$ for any $\lambda \in(0,1)$. Clearly, $z-x$ is an improvement direction at $x$; thus, $p(z-x)>0$ if $p$ is a preference gradient at $x$.

Lemma 3.8 Let $\succsim$ be a strictly convex and strictly monotonic, continuous weak order on X. Suppose also that $\succsim$ is differentiable at $x \in X$. Then the collection of $p \in \mathbb{R}_{++}^{n}$ that support $\mathcal{U}_{x}$ at $x$ properly is a singleton.

Proof. Observe first that the collection of preference gradients at $x$ is a subset of the collection of $p \in \mathbb{R}_{++}^{n}$ that support $\mathcal{U}_{x}$ at $x$ properly. This is an immediate implication of Lemmas 3.2 and 3.7. Hence, if $\succsim$ is differentiable at $x$, the collection of $p \in \mathbb{R}_{++}^{n}$ that support $\mathcal{U}_{x}$ at $x$ properly is non-empty.

We will prove now the contrapositive statement of the claim. To this end, suppose that $p, \widetilde{p} \in \mathbb{R}_{++}^{n}$ with $p \neq \widetilde{p}$ are such that both support $\mathcal{U}_{x}$ at $x$ properly. Let also $\bar{p}:=\lambda p+(1-\lambda) \widetilde{p}$ for some $\lambda \in(0,1)$, and consider the hyperplane $H_{\bar{p}, x}$. For any $z \in H_{\bar{p}, x}$ we have $\bar{p} z=\bar{p} x$. As both $p$ and $\widetilde{p}$ support $\mathcal{U}_{x}$ at $x$ properly, the latter equality implies that $x \succ z$ for any $z \in H_{\bar{p}, x} \backslash\{x\}$. The equality means also that at least one of $p z>p x$ and $\widetilde{p} z>\widetilde{p} x$ fails to hold. Take then $z \in H_{\bar{p}, x} \backslash\{x\}$ with $p(z-x)>0$. For $\mu \in(0,1)$ define also the point $z^{\mu}=x+\mu(z-x)$. As $z^{\mu} \in H_{\bar{p}, x} \backslash\{x\}$, we have $x \succ z^{\mu}$. This being moreover the case for any $\mu \in(0,1), z-x$ is a worsening direction at $x$. Yet we do have $p(z-x)>0$, and thus $p$ cannot be a preference gradient at $x$. And the same argument shows that $\widetilde{p}$ is not a preference gradient at $x$ either.

\subsection{Proof of Proposition 2}

Clearly, $\succsim$ is necessarily strictly convex and strictly monotonic (Proposition 1 ). Notice also that (iv) $\Rightarrow$ (i) obtains trivially while (iii) $\Rightarrow$ (iv) follows immediately from Lemma 3.6. That (ii) $\Rightarrow$ (iii), moreover, follows from Lemmas 3.8 and 3.4. We only need to show, therefore, that (i) $\Rightarrow$ (ii).

To argue ad absurdum, let $l_{i}(\cdot \mid x)$ be differentiable at $x_{-i}$ but suppose that $\succsim$ is not differentiable at $x$. Take $q_{-i} \in \mathbb{R}_{--}^{n-1}$ to be the unique subgradient of $l_{i}(\cdot \mid x)$ at $x_{-i}$. By Lemma 3.6, letting $q_{i}:=1 /\left(x_{i}-q_{-i} x_{-i}\right)$ 
and $p:=q_{i}\left(1,-q_{-i}\right) \in \mathbb{R}_{++}^{n}$ the latter vector supports uniquely $\mathcal{U}_{x}$ at $x$ (Lemma 3.4); it is thus the unique candidate for a preference gradient at that point (Lemma 3.7). Our hypothesis therefore is that $p$ is not a preference gradient of $\succsim$ at $x$; equivalently, that there exists $d \in \mathbb{R}^{n} \backslash\{0\}$ which is not an improvement direction for $\succsim$ at $x$ even though $p d>0$.

To arrive now at a contradiction, we consider the hyperplane on which we move when departing from $x$ in the direction of $d$. First, we establish that this hyperplane is given by a strictly positive vector (denoted by $\widetilde{p}\left(\rho_{0}\right)$ below), and supports properly locally at $x$ members of $\mathcal{I}_{x}$ that obtain when departing from $x$ in directions that are arbitrarily close to $d$ but put less weight on the dimensions in which $d$ is negative (i.e., when departing from $x$ in directions that approach $d$ from the northeast when $i=2=n$ ). Next, we observe that the weight on the dimensions in which $d$ is negative decreases with the distance from $x$ (i.e., the angle between $d$ and the direction in which the supported member of $\mathcal{I}_{x}$ lies shrinks as we approach $x$ from the northeast when $i=2=n$ ). This, however, leads to an impossibility. For, $q_{-i}$ being the unique subgradient of $l_{i}(\cdot \mid x)$ at $x_{-i}$, the hyperplane $H_{p, x}$ must fit between $H_{\widetilde{p}(\rho), x}$ and the supported members of $\mathcal{I}_{x}$; yet $p d>0$ necessitates that $H_{p, x}$ lies below $H_{\widetilde{p}(\rho), x}$ as we depart from $x$ in the direction of $d$.

Our formal argument proceeds in the above steps as follows.

Step 1. Let $\varepsilon_{0}>0$ be such that $\mathcal{B}_{\varepsilon_{0}}(x) \subset X$ while $l_{i}(\cdot \mid x)$ is strictly convex on $\mathcal{B}_{\varepsilon_{0}}(x)_{-i} \cap \mathcal{I}_{x}^{-i}$. Let also $\overline{r_{d}:=\varepsilon_{0}} /\|d\|$ so that $x+r d \in \mathcal{B}_{\varepsilon_{0}}(x)$ for any $r \in\left(0, r_{d}\right)$. Under the strict convexity of $\succsim$, the hypothesis that $d$ is not an improvement direction for $\succsim$ at $x$ necessitates that $x \succ x+r d$ for any $r \in\left(0, r_{d}\right)$. Moreover, $p \in \mathbb{R}_{++}^{n}$ and $p d>0$ together imply that $\mathcal{N}_{d}^{+} \neq \varnothing$. And since $x \succ x+r d$, by the strict monotonicity of $\succsim$, it must be also $\mathcal{N}_{d}^{-} \neq \varnothing$. Take then $i \in \mathcal{N}_{d}^{-}$and let $\widetilde{p}:=\left(p_{i} / 2, p_{-i}\right) \in \mathbb{R}_{++}^{n}$, which gives of course $(p-\widetilde{p}) d<0$. Define next the $[0,1] \rightarrow \mathbb{R}^{n}$ function $\widetilde{p}(\rho):=p-(1-\rho) \widetilde{p}$. Since $\widetilde{p}(0) d<0<\widetilde{p}(1) d$, there exists $\rho_{0} \in(0,1)$ that gives $\widetilde{p}\left(\rho_{0}\right) d=0$ where $\widetilde{p}\left(\rho_{0}\right)=\left(\left(1+\rho_{0}\right) p_{i} / 2, \rho_{0} p_{-i}\right) \in \mathbb{R}_{++}^{n}$. Define also the $\left(0, r_{d}\right) \times[0,1] \rightarrow \mathbb{R}_{++}^{n}$ function

$$
x(r, \mu)_{j}= \begin{cases}x_{j}+r d_{j} & j \in \mathcal{N} \backslash \mathcal{N}_{d}^{-} \\ x_{j}+(1-\mu) r d_{j} & j \in \mathcal{N}_{d}^{-}\end{cases}
$$

By the strict monotonicity of $\succsim$, and since $\mathcal{N} \backslash \mathcal{N}_{d}^{-} \supseteq \mathcal{N}_{d}^{+} \neq \varnothing$, this gives $x(r, 1) \succ x \succ x+r d=x(r, 0)$. Letting hence $u: X \rightarrow \mathbb{R}$ be a utility function for $\succsim$, we have $u(x(r, 0))<u(x)<u(x(r, 1))$ and the Intermediate-Value Theorem ensures the existence of $\mu_{r} \in(0,1)$ such that $u\left(x\left(r, \mu_{r}\right)\right)=u(x)$. By the strict monotonicity of $\succsim$, moreover, the mapping $r \mapsto \mu(r)=\mu_{r}$ is a function. And as

$$
\widetilde{p}\left(\rho_{0}\right)(x(r, \mu(r))-x)=r\left(\widetilde{p}\left(\rho_{0}\right) d-\sum_{j \in \mathcal{N}_{d}^{-}} \widetilde{p}\left(\rho_{0}\right)_{j} \mu(r) d_{j}\right)=-r \mu(r) \sum_{j \in \mathcal{N}_{d}^{-}} \widetilde{p}\left(\rho_{0}\right)_{j} d_{j},
$$

we have just established the existence of a function $\mu:\left(0, r_{d}\right) \rightarrow(0,1)$ such that $x(r, \mu(r)) \in \mathcal{I}_{x}$ with (13) satisfied everywhere on its domain.

Step 2. The function $\mu(\cdot)$ is strictly increasing everywhere on its domain. To show this arguing ad absurdum, let $r<r^{\prime}$ and suppose that $\mu(r) \geq \mu\left(r^{\prime}\right)$. Then, $1-\mu(r) \leq 1-\mu\left(r^{\prime}\right)$ and, by the strict monotonicity of $\succsim$, it must be $l_{i}\left(x\left(r, \mu\left(r^{\prime}\right)\right)_{-i} \mid x\right) \geq l_{i}\left(x(r, \mu(r))_{-i} \mid x\right)$. Moreover, the function $l_{i}(\cdot \mid x)$ 
being strictly convex, $r<r^{\prime}$ implies also that ${ }^{6}$

$$
\frac{l_{i}\left(x\left(r^{\prime}, \mu\left(r^{\prime}\right)\right)_{-i} \mid x\right)-l_{i}\left(x_{-i} \mid x\right)}{r^{\prime}}>\frac{l_{i}\left(x\left(r, \mu\left(r^{\prime}\right)\right)_{-i} \mid x\right)-l_{i}\left(x_{-i} \mid x\right)}{r}
$$

Putting these observations together, we get that

$$
\begin{aligned}
\left(1-\mu\left(r^{\prime}\right)\right) d_{i} & =\frac{x\left(r^{\prime}, \mu\left(r^{\prime}\right)\right)_{i}-x_{i}}{r^{\prime}} \\
& =\frac{l_{i}\left(x\left(r^{\prime}, \mu\left(r^{\prime}\right)\right)_{-i} \mid x\right)-l_{i}\left(x_{-i} \mid x\right)}{r^{\prime}} \\
& >\frac{l_{i}\left(x\left(r, \mu\left(r^{\prime}\right)\right)_{-i} \mid x\right)-l_{i}\left(x_{-i} \mid x\right)}{r} \\
& \geq \frac{l_{i}\left(x(r, \mu(r))_{-i} \mid x\right)-l_{i}\left(x_{-i} \mid x\right)}{r}=(1-\mu(r)) d_{i}
\end{aligned}
$$

which is, of course, absurd given that $d_{i}<0$.

Step 3. Observe next that, $q_{-i}$ being the gradient at $x_{-i}$ of the (locally) convex function $l_{i}(\cdot \mid x)$, for any direction $v_{-i} \in \mathbb{R}^{n-1}$ the quantity

$$
\left[l_{i}\left(x_{-i}+\varepsilon v_{-i} \mid x\right)-x_{i}\right] / \varepsilon=\left[l_{i}\left(x_{-i}+\varepsilon v_{-i} \mid x\right)-l_{i}\left(x_{-i} \mid x\right)\right] / \varepsilon, \quad \varepsilon \in\left(0, \varepsilon_{0} /|| v_{-i}||\right)
$$

approaches $q_{-i} v_{-i}$ from above as $\varepsilon \searrow 0 .^{7}$ Formally,

$$
\forall \Delta>0, \exists \varepsilon_{\Delta} \in\left(0, \varepsilon_{0} / \| v_{-i}||\right): l_{i}\left(x_{-i}+\varepsilon v_{-i} \mid x\right)-x_{i}<\varepsilon\left(\Delta+q_{-i} v_{-i}\right) \quad \forall \varepsilon \in\left(0, \varepsilon_{\Delta}\right)
$$

Let then $v_{-i}=d_{-j}$ and $\Delta:=-\left(1-\rho_{0}\right)\left(1-\mu\left(r_{d} / 2\right)\right) d_{i} /\left(2 \rho_{0}\right)$. Since $r_{d}=\varepsilon_{0} /\|d\| \leq \varepsilon_{0} /\left\|d_{-i}\right\|$, there exists $\varepsilon_{1} \in\left(0, r_{d} / 2\right)$ such that, for all $\varepsilon \in\left(0, \varepsilon_{1}\right)$,

$$
\begin{aligned}
\rho_{0} q_{i}\left(x(\varepsilon, \mu(\varepsilon))_{i}-x_{i}\right) & =\rho_{0} q_{i}\left(l_{i}\left(x(\varepsilon, \mu(\varepsilon))_{-i} \mid x\right)-x_{i}\right) \\
& \leq \rho_{0} q_{i}\left(l_{i}\left(x(\varepsilon, 0)_{-i} \mid x\right)-x_{i}\right) \\
& =\rho_{0} q_{i}\left(l_{i}\left(x_{-i}+\varepsilon d_{-i} \mid x\right)-x_{i}\right) \\
& <\varepsilon \rho_{0} q_{i}\left(\Delta+q_{-i} d_{-i}\right) \\
& =-\left(\frac{1-\rho_{0}}{2}\right) q_{i}\left(1-\mu\left(\frac{r_{d}}{2}\right)\right) \varepsilon d_{i}+\rho_{0} q_{i} q_{-i} \varepsilon d_{-i} \\
& <-\left(\frac{1-\rho_{0}}{2}\right) q_{i}(1-\mu(\varepsilon)) \varepsilon d_{i}+\rho_{0} q_{i} q_{-i} \varepsilon d_{-i} \\
& =-\left(\frac{1-\rho_{0}}{2}\right) q_{i}(1-\mu(\varepsilon)) \varepsilon d_{i}+(1-\mu(\varepsilon)) \rho_{0} q_{i} \sum_{j \in \mathcal{N}_{d}^{-} \backslash\{i\}} q_{j} \varepsilon d_{j}+\rho_{0} q_{i} \sum_{j \in \mathcal{N}_{d}^{+}} q_{j} \varepsilon d_{j} \\
& +\mu(\varepsilon) \rho_{0} q_{i} \sum_{j \in \mathcal{N}_{d}^{-} \backslash\{i\}} q_{j} \varepsilon d_{j} \\
& =\left(\frac{1-\rho_{0}}{2}\right) p_{i}\left(x_{i}-x(\varepsilon, \mu(\varepsilon))_{i}\right)+\widetilde{p}\left(\rho_{0}\right)_{-i}\left(x_{-i}-x(\varepsilon, \mu(\varepsilon))_{-i}\right) \\
& -\varepsilon \mu(\varepsilon) \sum_{j \in \mathcal{N}_{d}^{-} \backslash\{i\}} \widetilde{p}\left(\rho_{0}\right)_{j} d_{j},
\end{aligned}
$$

${ }^{6}$ Given $K \in \mathbb{N} \backslash\{0\}$ and a strictly convex function $f: S \rightarrow \mathbb{R}$ defined on an open and convex set $S \subseteq \mathbb{R}^{K}$, a vector $v \in \mathbb{R}^{K}$, and $\varepsilon \in \mathbb{R} \backslash\{0\}$, the ratio $[f(x+\varepsilon v)-f(x)] / \varepsilon$ is a strictly increasing function of $\varepsilon$ (see Theorem 6.2.15 in de la Fuente (2000)).

${ }^{7}$ The limit as $\varepsilon \searrow 0$ of the quantity in (14) above is the directional derivative of $l_{i}(\cdot \mid x)$ at at $x_{-i}$ in the direction of the vector $v_{-i}$. The limit exists because the function is convex on $\mathcal{B}_{\varepsilon_{0}}(x)^{-i} \cap \mathcal{I}_{x}^{-i}$; it coincides with the quantity $q_{-i} v_{-i}$ because the function is in addition differentiable at $x_{-i}$ (see Section 4 in the Online Appendix for more details). That the quantity in (14) approaches the limit from above follows from the observation in the preceding footnote. 
where the first and third inequalities above are due to the monotonicity of $\succsim$ and $\mu(\cdot)$, respectively. For any $\varepsilon \in\left(0, \varepsilon_{1}\right)$, therefore, we have

$$
\begin{aligned}
\varepsilon \mu(\varepsilon) \sum_{j \in \mathcal{N}_{d}^{-} \backslash\{i\}} \widetilde{p}\left(\rho_{0}\right)_{j} d_{j} & <\left(\frac{1+\rho_{0}}{2}\right) p_{i}\left(x_{i}-x(\varepsilon, \mu(\varepsilon))_{i}\right)+\widetilde{p}\left(\rho_{0}\right)_{-i}\left(x_{-i}-x(\varepsilon, \mu(\varepsilon))_{-i}\right) \\
& =\widetilde{p}\left(\rho_{0}\right)(x-x(\varepsilon, \mu(\varepsilon)))=\varepsilon \mu(\varepsilon) \sum_{j \in \mathcal{N}_{d}^{-}} \widetilde{p}\left(\rho_{0}\right)_{j} d_{j},
\end{aligned}
$$

the last equality due to (13). This implies though that $\widetilde{p}\left(\rho_{0}\right)_{i} d_{i}>0$, which is, of course, absurd.

The following result supports parts of the argument in the proofs for Propositions 3-4. In what follows, we say that a weak order $\succsim$ on $X$ is weakly $C^{1}$ at $x \in X$ if $\mathcal{I}_{x}$ is a $C^{1}$ manifold of dimension $n-1$ locally at $x$, i.e. if there exists $\varepsilon>0$ such that $\mathcal{B}_{\varepsilon}(x) \cap \mathcal{I}_{x}$ is a $C^{1}$ manifold of dimension $n-1$. Recall also that a function $f: A \rightarrow \mathbb{R}$ ( $A \subseteq \mathbb{R}^{k}$ open) is continuously differentiable (equivalently, $C^{1}$ ) at $x \in A$ if it is continuously differentiable on $\mathcal{B}_{\mathcal{\varepsilon}}(x) \subset A$ for some $\varepsilon>0$.

Lemma 3.9 Let $\succsim$ be a strictly convex, strictly monotonic, continuous weak order on $X$. For any $(x, z, i) \in$ $X \times \mathcal{I}_{x} \times \mathcal{N}, \succsim$ is weakly $C^{1}$ at $z$ if and only if $l_{i}(\cdot \mid x)$ is $C^{1}$ at $z_{-i}$.

Proof. Take arbitrary $x \in X$ and $i \in \mathcal{N}$. For the "if" direction, let $l_{i}(\cdot \mid x)$ be $C^{1}$ at $z_{-i}$ and observe that the graph of a $C^{r}$ function $f: A \rightarrow \mathbb{R}^{m}\left(A \subseteq \mathbb{R}^{k}\right.$ open, $r \in \mathbb{N}$ ) is a $C^{r}$ (n-dimensional) manifold -see Section 1.H.1 in Mas-Colell (1985). The graph of $l_{i}(\cdot \mid x)$ being the indifference set $\mathcal{I}_{x}$, the latter is clearly a $C^{1}$ manifold at $z$.

For the "only if," let $\mathcal{I}_{x}$ be a $C^{1}$ manifold at $z$. There exists $\epsilon>0$ and a $C^{1}$ regular function $\xi: \mathcal{B}_{\epsilon}(z) \times$ $\mathcal{B}_{\epsilon}(z) \rightarrow \mathbb{R}$ such that $\mathcal{B}_{\epsilon}(z) \cap \mathcal{I}_{x}=\xi^{-1}(0)$ (see for instance Section 1.H.2 in Mas-Colell (1985)). It follows then from the implicit-function theorem that the mapping $l_{i}(\cdot \mid x)$ is a well-defined $C^{1}$ regular function on $\mathcal{B}_{\epsilon_{0}}(z) \cap \mathcal{I}_{x}^{-i}$ for some $\epsilon_{0} \in(0, \epsilon)$.

\subsection{Proof of Proposition 3}

In light of Lemma 3.9 that (iii) $\Rightarrow$ (i) follows from Proposition 2. To show next that (ii) $\Rightarrow$ (iii) observe that, $x(\cdot)$ being injective, $p(\cdot)$ is a function. For any $(x, z, i) \in X \times \mathcal{I}_{x} \times \mathcal{N}$, therefore, $l_{i}(\cdot \mid x)$ is differentiable at $z_{-i}$ (Proposition 2 ) with the gradient defined from the singleton $p(z)$ by equation (12):

$$
\nabla l_{i}\left(z_{-i} \mid x\right)=-p_{-i}(z) / p_{i}(z)
$$

Moreover, since $x(\cdot)$ is in fact an homeomorphism, $p(\cdot)$ is continuous at $z$ so that $l_{i}(\cdot \mid x)$ is $C^{1}$ at $z_{-i}$. The claim follows once again from Lemma 3.9.

To establish finally that (i) $\Rightarrow$ (ii), we will make use of the following result.

Lemma 3.10 Let the onto demand function $x: Y \rightarrow X$ for some $Y \subseteq \mathbb{R}_{++}^{n}$ be generated by the continuous weak order $\succsim$ on $X$. For any $p \in Y, x(\cdot)$ is continuous at $p$ if $\succsim$ is differentiable at $x(p)$.

Proof. Observe first that $\succsim$ is necessarily strictly convex and strictly monotonic (Proposition 1 ). Take an arbitrary $p \in Y$ and let $x=x(p)$. Choose also $\epsilon>0$ sufficiently small so that $\mathcal{B}_{\epsilon}(x) \subset X$. Consider the problem $\max _{\succsim}\left\{z \in \mathrm{cl}\left(\mathcal{B}_{\epsilon / 2}(x)\right): p^{\prime} z \leq 1\right\}$ for $p^{\prime} \in \mathbb{R}_{++}^{n}$. By Proposition 1, there exists $\widetilde{Y} \subseteq \mathbb{R}_{++}^{n}$ and an 
onto solution function $\widetilde{x}: \widetilde{Y} \rightarrow \mathcal{B}_{\epsilon / 2}(x)$. For any $p^{\prime} \in \tilde{Y}$, moreover, the constrained set is compact - being but the intersection of the compact sets $\mathrm{cl}\left(\mathcal{B}_{\epsilon / 2}(x)\right)$ and $\left\{z \in \mathbb{R}_{+}^{n}: p^{\prime} z \leq 1\right\}$. Thus, by Berge's Maximum theorem (see for instance Theorems 7.2.1-7.2.2 in de la Fuente (2000)), $\widetilde{x}(\cdot)$ is continuous on $\widetilde{Y}$ - which is open since the function is onto an open set.

We will show next that $\widetilde{Y} \subseteq Y$ while $\widetilde{x}(\cdot)$ is the restriction of $x(\cdot)$ on $\widetilde{Y}$. To this end, observe that the arbitrary $p^{\prime} \in \widetilde{Y}$ supports $\mathcal{U}_{\widetilde{x}\left(p^{\prime}\right)}$ at $\widetilde{x}\left(p^{\prime}\right)$. For if there exists $z \in \mathcal{U}_{\widetilde{x}\left(p^{\prime}\right)}$ with $p^{\prime} z \leq p^{\prime} \widetilde{x}\left(p^{\prime}\right)$, then any $\lambda \in(0,1)$ would give $z^{\lambda}=\lambda z+(1-\lambda) \widetilde{x}\left(p^{\prime}\right) \succ \widetilde{x}\left(p^{\prime}\right)$ while $p^{\prime} z^{\lambda} \leq 1$. As though $\lim _{\lambda \rightarrow 0} z^{\lambda}=\widetilde{x}\left(p^{\prime}\right)$, for any $\varepsilon>0$, we have that $z^{\lambda} \in \mathcal{B}_{\varepsilon}\left(\widetilde{x}\left(p^{\prime}\right)\right)$ for sufficiently small $\lambda$. Yet, a small enough $\varepsilon$ ensures that $\mathcal{B}_{\varepsilon}\left(\tilde{x}\left(p^{\prime}\right)\right) \subset \mathcal{B}_{\epsilon / 2}(x)$, contradicting the optimality of $\widetilde{x}\left(p^{\prime}\right)$. Recall now Lemma 3.1. The fact that $p^{\prime}$ supports $\mathcal{U}_{\widetilde{x}\left(p^{\prime}\right)}$ at $\widetilde{x}\left(p^{\prime}\right)$ implies that $\widetilde{x}\left(p^{\prime}\right) \in \max _{\succsim}\left\{x \in X: p^{\prime} x \leq 1\right\}$. As the latter set though is the singleton $x\left(p^{\prime}\right)$ while $x(\cdot)$ is onto, it cannot but be $\widetilde{x}\left(p^{\prime}\right)=x\left(p^{\prime}\right)$ and $p^{\prime} \in Y$.

Notice finally that, since $\widetilde{x}(\cdot)$ is onto $\mathcal{B}_{\epsilon / 2}(x)$ and coincides on its domain with $x(\cdot)$, there exists $p^{\prime \prime} \in \tilde{Y}$ such that $\widetilde{x}\left(p^{\prime \prime}\right)=x=x\left(p^{\prime \prime}\right)$. However, $\succsim$ being differentiable at $x$, we cannot have $x\left(p^{\prime \prime}\right)=x=x(p)$ unless $p^{\prime \prime}=p$ (recall Proposition 2). This establishes that $\widetilde{Y}=Y$ while $\tilde{x}(\cdot)$ coincides with $x(\cdot)$. The claim now follows.

By the preceding lemma, for any $x \in X$, if $\succsim$ is differentiable at $x$ then $x(\cdot)$ is continuous at the unique (recall Proposition 2) $p \in Y: x=x(p)$. Given this and the Invariance of Domain Theorem, the claim follows. ${ }^{8}$

\subsection{Proof of Proposition 4}

In light of Proposition 3, we need to show that (ii) $\Leftrightarrow$ (iii) With respect to (iii) $\Rightarrow$ (ii), by the implicit function theorem (or by Lemma 5.3 in the Online Appendix), $u(\cdot)$ being $C^{1}$ at $x$ implies that $l_{i}(\cdot \mid x)$ is also $C^{1}$ at $x_{-i}$ for any $i \in \mathcal{N}$. The claim then is due to Lemma 3.9. It remains thus to establish that (ii) $\Rightarrow$ (iii).

Homothetic preferences. As is well-known, a strictly convex, strictly monotonic, continuous and homothetic weak order $\succsim$ on $X \subseteq \mathbb{R}_{++}^{n}$ admits a continuous, strictly monotonic, strictly quasiconcave and homogenous of degree one utility representation $u: X \rightarrow \mathbb{R}_{++}{ }^{9}$ Given these properties, $u(\cdot)$ is in fact concave on $X .{ }^{10}$ The claim is due to the following result.

Lemma 3.11 Let the strictly convex and strictly monotonic continuous weak order $\succsim$ on $X$ be represented by the concave utility function $u: X \rightarrow \mathbb{R}$. For any $(x, z, i) \in X \times \mathcal{I}_{x} \times \mathcal{N}, u(\cdot)$ is $C^{1}$ at $z$ if and only if $\succsim$ is weakly $C^{1}$ at $z$.

Proof. For the "only if" direction, recall the Implicit Function Theorem (or Lemma 5.3 in the Online Appendix): if $u(\cdot)$ is $C^{1}$ at $z$ then $l_{i}(\cdot \mid x)$ is also $C^{1}$ at $z_{-i}$. The claim follows from Lemma 3.9. For the

\footnotetext{
${ }^{8}$ Letting $A, B \subseteq \mathbb{R}^{n}$ with $A$ open, the Invariance of Domain Theorem - see Brouwer (1912) - states that a function $f: A \rightarrow B$ being injective and continuous suffices for it to be an homeomorphism and for $f(A)$ to be open.

${ }^{9}$ Letting $A \subseteq \mathbb{R}_{+}^{n}$, a strictly monotonic and homogenous of degree one function $f: A \rightarrow \mathbb{R}$ gives $f(x)<f(\lambda x)=\lambda f(x)$, for any $\lambda>1$ and any $x \in A \backslash\{0\}$. Clearly, it must be that $f(x)>0$ for any $x \in A \cap \mathbb{R}_{++}^{n}$.

${ }^{10}$ See Theorem 1 in Prada (2011): letting $A \subseteq \mathbb{R}_{+}^{n}$, if a function $f: A \rightarrow \mathbb{R}$ is quasiconcave, increasing and homogenous of degree $\gamma$ with $0<\gamma \leq 1$ then it is concave.
} 
"if" direction, observe first that $u(\cdot)$ being concave, $-u(\cdot)$ is convex. The subdifferential $\partial(-u(z))$ therefore will be non-empty. Yet any $-p \in \partial(-u(z))$ gives $u(z)-u\left(z^{\prime}\right) \geq p\left(z-z^{\prime}\right)$ for any $z^{\prime} \in X$; i.e., $0 \leq u\left(z^{\prime}\right)-u(z) \leq p\left(z^{\prime}-z\right)$ for any $z^{\prime} \in \mathcal{U}_{z}$. That is, any $p \in \partial(u(z))$ supports $\mathcal{U}_{z}$ at $z$, which implies in turn that $\partial(u(z)) \subset Y$ (see Lemmas 3.2 and 3.4). By Proposition 3 then, $\succsim$ is weakly $C^{1}$ at $z$ only if $\partial(u(z))$ is a singleton and $\partial(u(\cdot))=p(\cdot)$ is continuous at $z$.

Quasi-linear preferences. A quasilinear, strictly convex and strictly monotonic continuous weak order on $X$ is represented by a utility function $u: X \rightarrow \mathbb{R}$ given by

$$
u(x)=x_{i}+v\left(x_{-i}\right), \quad i \in \mathcal{N}
$$

for some continuous, strictly increasing and strictly quasiconcave function $v: X_{-i} \rightarrow \mathbb{R}$. Hence, $l_{i}(\cdot \mid x)=$ $x_{i}+v\left(x_{-i}\right)-v(\cdot)$ and the claim follows immediately from Lemma 3.9. ${ }^{11}$

\section{References}

AfriAt, S. N. (2014): Demand Functions and the Slutsky Matrix, Princeton: Princeton University Press.

Aguiar, V. H., P. HJertstrand, And R. Serrano (2020): “A Rationalization of the Weak Axiom of Revealed Preference," working paper.

Aliprantis, C. D. AND K. C. Border (2006): Infinite Dimensional Analysis, 3rd edition, Berlin Heidelberg: Springer.

AMIR, R., P. ERICKSON, AND J. JIN (2017): “On the Microeconomic Foundations of Linear Demand for Differentiated Products," Journal of Economic Theory, 169, 641-665.

Berry, S., A. GHANDhI, AND P. Haile (2013): “Connected Substitutes and Invertibility of Demand," Econometrica, 81, 2087-2111.

BilanCINI, E. AND L. Boncinelli (2010): "Single-valuedness of the Demand Correspondence and Strict Convexity of Preferences: An Equivalence Result," Economics Letters, 108, 299-302.

BrouWer, L. (1912): “Invariantz des n-Dimensionalen Gebiets,” Mathematische Annalen, 71, 305-315.

CHENG, L. (1985): “Inverting Systems of Demand Functions," Journal of Economic Theory, 37, 202-210.

CHIAPPORI, P.-A. AND J.-C. Rochet (1987): “Revealed Preferences and Differentiable Demand,” Econometrica, 55, 687-691.

DE LA Fuente, A. (2000): Mathematical Methods and Models for Economists, Cambridge: Cambridge University Press, 3rd ed.

DebreU, G. (1972): “Smooth Preferences," Econometrica, 40, 603-615.

\footnotetext{
${ }^{11}$ We note that a fourth equivalent statement can be added to Proposition 4 in the quasilinear-preference case covered by this result: $\succsim$ is representable by a differentiable utility function. This is so because the non-linear component $v\left(x_{-i}\right)$ of such a utility function evaluated at some bundle coincides with the value of the indifference-projection function $l_{i}\left(x_{-i} \mid x\right)$.
} 
Gale, D. AND H. NiKaido (1965): “The Jacobian Matrix and Global Univalence of Mappings," Mathematische Annalen, 159, 81-93.

Hildenbrand, W. (1994): Market Demand: Theory and Empirical Evidence, Princeton: Princeton University Press.

Hurwicz, L. AND H. UzaWA (1971): “On the Integrability of Demand Functions," in Preferences, Utility and Demand, ed. by J. S. Chipman, L. Hurwicz, M. K. Richter, and H. F. Sonnenschein, New York: Harcourt Brace Jovanovich, 114-148.

JeRISON, M. AND J. K.-H. QuAH (2008): "Law of Demand," in The New Palgrave Dictionary of Economics, ed. by S. N. Durlauf and L. E. Blume, Palgrave, 2nd ed.

Kannai, Y. (1989): "A Characterization of Monotone Demand Functions," Journal of Mathematical Economics, 18, 87-94.

KATZNER, D. W. (1970): Static Demand Theory, New York: Macmillan.

KÜBleR, F. AND H. PolemarChaKIS (2017): “The Identification of Beliefs from Asset Demand," Econometrica, 85, 1219-1238.

MAS-COLELL, A. (1985): The Theory of General Equilibrium: A Differentiable Approach, Econometric Society Monographs, Cambridge University Press.

Mas-Colell, A., M. D. Whinston, And J. R. Green (1995): Microeconomic Theory, New York: Oxford University Press.

MATZKIN, R. L. AND M. K. RiCTHER (1991): “Testing Strictly Concave Rationality,” Journal of Economic Theory, 53, 287-303.

Mitjuschin, L. G. And W. M. Polterovich (1978): “Criteria for Monotonicity of Demand Functions," Ekonomika i Mathematicheskii Metody, 14, 122-128, (in Russian).

NeILSON, W. S. (1991): “Smooth Indifference Sets,” Journal of Mathematical Economics, 20, 181-197.

PradA, J. D. (2011): “A Note on Concavity, Homogeneity, and Non-Increasing Returns to Scale," Economics Bulletin, 31, 100-105.

RENOU, L. AND K. H. SCHLAG (2014): “Ordients: Optimization and Comparative Statics without Utility Functions," Journal of Economic Theory, 154, 612-632.

Rubinstein, A. (2006): Lecture Notes in Microeconomic Theory: The Economic Agent, Princeton: Princeton University Press. 